\title{
Deep Learning-Based Intrusion Detection for Distributed Denial of Service Attack in Agriculture 4.0
}

\author{
Mohamed Amine Ferrag ${ }^{1}{ }^{(}$, Lei Shu ${ }^{2,3, *}$, , Hamouda Djallel ${ }^{1} \mathbb{D}$ and Kim-Kwang Raymond Choo ${ }^{4}$ \\ 1 Department of Computer Science, Guelma University, Guelma 24000, Algeria; \\ ferrag.mohamedamine@univ-guelma.dz (M.A.F.); hamouda.djallel@univ-guelma.dz (H.D.) \\ 2 College of Artificial Intelligence, Nanjing Agricultural University, Nanjing 210031, China \\ 3 School of Engineering, University of Lincoln, Lincoln LN6 7TS, UK \\ 4 Department of Information Systems and Cyber Security, University of Texas at San Antonio, \\ San Antonio, TX 78249, USA; raymond.choo@fulbrightmail.org \\ * Correspondence: lei.shu@ieee.org
}

Citation: Ferrag, M.A.; Shu, L.; Djallel, H.; Choo, K.-K.R. Deep Learning-Based Intrusion Detection for Distributed Denial of Service Attack in Agriculture 4.0. Electronics 2021, 10, 1257. https://doi.org/ 10.3390/electronics10111257

Academic Editor: Khaled Elleithy

Received: 4 May 2021

Accepted: 19 May 2021

Published: 25 May 2021

Publisher's Note: MDPI stays neutral with regard to jurisdictional claims in published maps and institutional affiliations.

Copyright: (C) 2021 by the authors. Licensee MDPI, Basel, Switzerland. This article is an open access article distributed under the terms and conditions of the Creative Commons Attribution (CC BY) license (https:// creativecommons.org/licenses/by/ $4.0 /)$.

\begin{abstract}
Smart Agriculture or Agricultural Internet of things, consists of integrating advanced technologies (e.g., NFV, SDN, 5G/6G, Blockchain, IoT, Fog, Edge, and AI) into existing farm operations to improve the quality and productivity of agricultural products. The convergence of Industry 4.0 and Intelligent Agriculture provides new opportunities for migration from factory agriculture to the future generation, known as Agriculture 4.0. However, since the deployment of thousands of IoT based devices is in an open field, there are many new threats in Agriculture 4.0. Security researchers are involved in this topic to ensure the safety of the system since an adversary can initiate many cyber attacks, such as DDoS attacks to making a service unavailable and then injecting false data to tell us that the agricultural equipment is safe but in reality, it has been theft. In this paper, we propose a deep learning-based intrusion detection system for DDoS attacks based on three models, namely, convolutional neural networks, deep neural networks, and recurrent neural networks. Each model's performance is studied within two classification types (binary and multiclass) using two new real traffic datasets, namely, CIC-DDoS2019 dataset and TON_IoT dataset, which contain different types of DDoS attacks.
\end{abstract}

Keywords: deep learning approaches; intrusion detection system; Agriculture 4.0; DDoS attack; smart agriculture

\section{Introduction}

The 4th revolution of the industrial era (or Industry 4.0) is the new industry trend that defines the Smart Factory concept [1]. This concept is based on emerging technologies such as Fog computing, Cloud computing, Artificial Intelligence, Deep learning... etc. To provide an optimization of operations and reduction of costs, these technologies are employed to establish a connection between machines and the Internet, through the Internet-of-Things, to collect information in the Cloud and Edge and then process them using artificial intelligence algorithms. Industry 4.0 is expected to transform the agricultural industry and advance the 4th agricultural revolution, known as Agriculture 4.0. The first three industrial revolutions deeply reshaped the agricultural industries from indigenous agriculture (Agriculture 1.0) towards mechanized agriculture (Agriculture 2.0) and recent precision farming (Agriculture 3.0), as presented in Figure 1 [2,3].

In the most recent years, the IoT application has been deployed for Agriculture 4.0 using wireless sensor networks such as, Supply chain management, Smart monitoring, Smart water, Agrochemicals applications, Disease management, and Smart harvesting. Figure 2 presents the IoT, IIoT, Industry 4.0, Agriculture 4.0 and the common concepts. Industry 4.0 focuses primarily on the manufacturing sector, Agriculture 4.0 focuses on the Agriculture sector, whereas IIoT covers all sectors where industrial/professional equipment 
is used. However, with thousands of IoT-based devices deployed in the open field, there are many new cyber security threats in Agriculture 4.0. When an adversary attempting to penetrate Agriculture 4.0 network, it use several different approaches such as DDoS attacks, scanning attacks, false data injection attacks, to disrupt the functioning of the IoT-based devices. For example, in the soil $\mathrm{pH}$ parameters, if the $\mathrm{pH}$ rises excessively, it means that the farmer will increase ammonium input, and if the $\mathrm{pH}$ falls, it indicates that the farmer will reduce ammonium input. With this information, an adversary can launch DDoS attacks to disrupt the $\mathrm{pH}$ parameters. Hence, this private information (i.e., the $\mathrm{pH}$ parameters) must be protected from cyber attacks [4]. To protect Agriculture 4.0 from destruction, change, unauthorized access, or attack, Security researchers propose the use of an intrusion detection system (IDS) beside the authentication, access control, and integrity techniques [5-7].

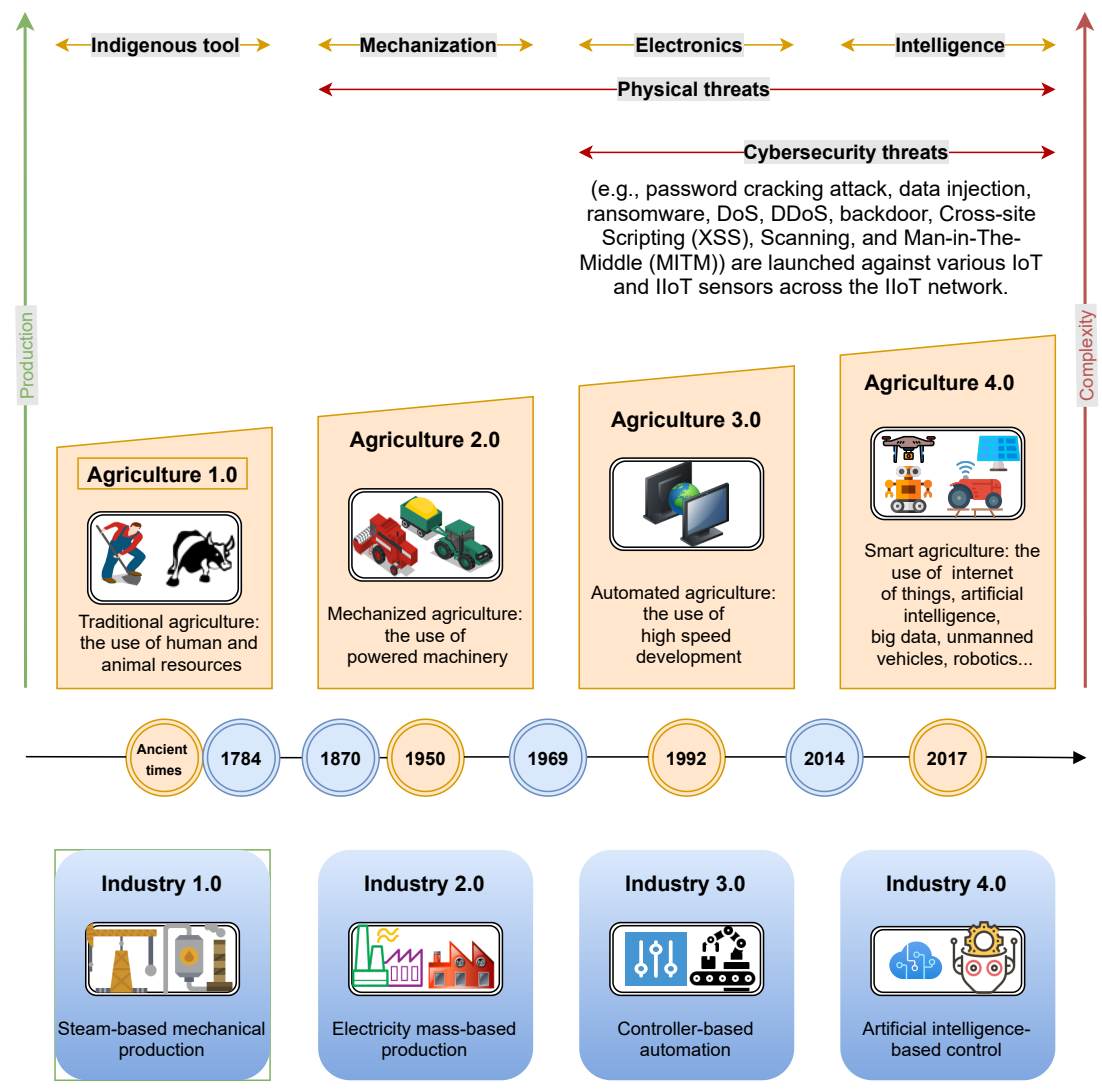

Figure 1. Agricultural revolutions with industrial revolutions and related cyber security threats.

The IDS system is a mechanism monitoring the network traffic, which is used to detect suspicious or abnormal activities and then enables preventive measure on the intrusion risks. Therefore, intrusion detection systems can be divided into two major types, namely, (1) Network Intrusion Detection Systems (NIDS) and (2) Host Intrusion Detection Systems (HIDS). The NIDS is typically deployed or located at critical network points to ensure that it covers the locations where the traffic is more susceptible to attacked, while the HIDS systems works on any device on the network that has Internet access. To detecting intrusion, there are two main techniques, namely, IDS (1) based on anomalies and (2) IDS based on signatures [8]. The signature-based IDS (i.e., Misuse Detection or Knowledgebased Detection) concentrates on identifying a "signature", patterns of intrusion event, and it is as efficient as updating the database at a specific moment of time. Based on monitoring regular activities, the anomaly-based IDS (i.e., Behavior-based Detection) uses machine learning techniques to compare trustworthy behavioral patterns with new behaviors. When an administrator receives an alert via the IDS system, it uses Intrusion Prevention Systems (IPS) to block the threat such as Trojan horse, DDoS attacks, etc. [9]. 


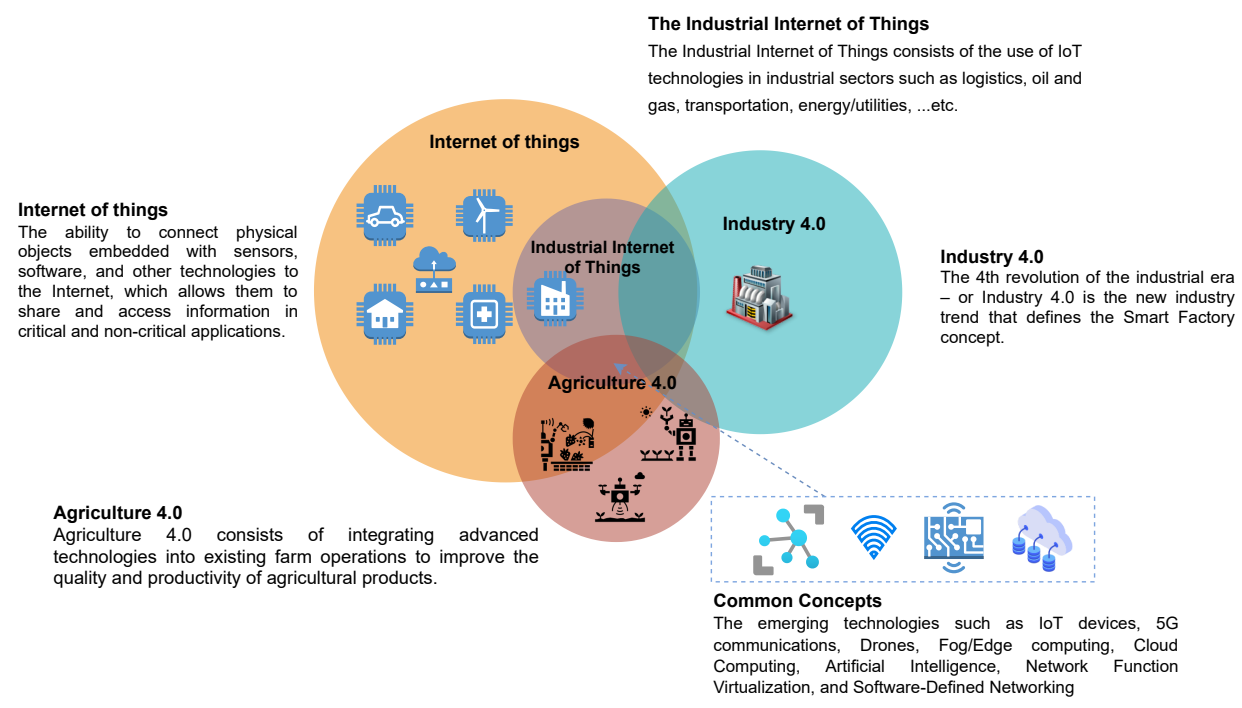

Figure 2. IoT, IIoT, Industry 4.0, Agriculture 4.0 and the common concepts.

This paper focuses on developing and employing deep-learning approaches for detecting cyber threats (i.e., anomaly-based IDS). There are some recently proposed IDS systems that employ deep learning strategies for IoT applications, such as wireless networks [10], big data environments [11], industrial cyber-physical systems [12], SCADA systems [13], smart grids [14], internet of vehicles [15], and cloud computing [16]. Deep learning approaches are also used in Agriculture 4.0, for crop hail damage, soil and vegetation/crop mapping, crop monitoring, irrigation, greenhouse monitoring, etc. [17]. However, there are eight big challenges in the field of intrusion detection systems for Agriculture 4.0: (1) Data collection that contains IIoT traffics with cyber attacks, (2) Less amount of training data, (3) Non-representative training data, (4) Poor quality of data, (5) Irrelevant/unwanted features, (6) Overfitting the training data, (7) Underfitting the training data, (8) Offline learning \& deployment of the model [18]. Our proposed model overcomes these challenges. The datasets used in our paper are very popular, recent, and used by the scientific community for developing intrusion detection systems for IloT networks.

Our contributions in this work are:

- We propose three deep learning-based IDS models, including a convolutional neural network-based IDS model,a deep neural network-based IDS model, and a recurrent neural network-based IDS model.

- We provide a performance evaluation and comparative analysis of machine learning and deep learning approaches for cyber security in agriculture 4.0.

- We review three models of deep learning; namely, convolutional neural networks, deep neural networks, and recurrent neural networks. Each model's performance is studied within two classification types (binary and multiclass) using two new real traffic datasets, namely, CIC-DDoS2019 dataset and TON_IoT dataset .

- We focus on the following important performance indicators: false alarm rate (FAR), precision, F-score, detection rate (DR), recall, True Negative Rate (TNR), False Accept Rate (FAR), ROC Curve, and accuracy.

The rest of this work is structured as follows. Section 2 review the related works. Section 3 presents the implementation of IDSs. Section 4 provides a comparative study on Deep Learning based-IDS for Agriculture 4.0. Lastly, Section 5 presents conclusions.

\section{Related Work}

The popularity of deep learning in different fields of Big Data has created a lot of attention in the area of cyber security. According to its architectural conception, deep learning can be categorized in various types, such as generative and discriminative [19]. The intrusion detection systems have been investigated with the use of shallow and deep 
networks to identify anomalous patterns in both network and host-based systems. The summary of deep learning approaches for network intrusion detection for the IoT networks is presented in Table 1.

Table 1. Summary of deep learning approaches for network intrusion detection for the IoT networks.

\begin{tabular}{|c|c|c|c|c|c|c|}
\hline System & Year & Network Model & Deep Learning Techniques & The Basic Idea & Dataset Used & Performance Metrics \\
\hline $\begin{array}{l}\text { Diro and Chil- } \\
\text { amkurti [20] }\end{array}$ & 2018 & Social internet of things & $\begin{array}{l}\text { Deep learning approach } \\
\text { with softmax as activation } \\
\text { function }\end{array}$ & $\begin{array}{l}\text { Deploy the distributed attack detection } \\
\text { system at the fog computing layer }\end{array}$ & $\begin{array}{l}\text { NSL-KDD, ISCX, and } \\
\text { KDDCUP99 }\end{array}$ & $\begin{array}{l}\text { Accuracy, detection rate, } \\
\text { and false alarm rate }\end{array}$ \\
\hline Muna et al. [21] & 2018 & Industrial internet of things & $\begin{array}{l}\text { Unsupervised deep auto- } \\
\text { encoder algorithm }\end{array}$ & $\begin{array}{l}\text { The unsupervised deep auto-encoder al- } \\
\text { gorithm is used to learn normal network } \\
\text { behaviors, while a standard supervised } \\
\text { deep neural network model is used to } \\
\text { classify network behaviors }\end{array}$ & $\begin{array}{l}\text { NSL-KDD and UNSW- } \\
\text { NB15 }\end{array}$ & $\begin{array}{l}\text { Accuracy, detection rate, } \\
\text { and false positive rate }\end{array}$ \\
\hline $\begin{array}{l}\text { HaddadPajouh et } \\
\text { al. [22] }\end{array}$ & 2018 & Internet of things & $\begin{array}{l}\text { Deep Recurrent Neural } \\
\text { Network }\end{array}$ & $\begin{array}{l}\text { Detecting IoT malware based on three } \\
\text { stages, namely, collection data, feature } \\
\text { extrication, and deep threat classifier }\end{array}$ & IoT malware dataset & Accuracy, detection rate \\
\hline $\begin{array}{l}\text { Vinayakumar et al. } \\
\text { [23] }\end{array}$ & 2020 & $\begin{array}{l}\text { The Internet of Things net- } \\
\text { works of smart cities }\end{array}$ & $\begin{array}{l}\text { Cost-sensitive model- } \\
\text { based deep learning, }\end{array}$ & $\begin{array}{l}\text { Uses a two-tier environment for moni- } \\
\text { toring DNS logs }\end{array}$ & AmritaDGA & $\begin{array}{l}\text { F1-score, true positive rate, } \\
\text { False positive rate, preci- } \\
\text { sion, accuracy, recall }\end{array}$ \\
\hline Parra et al. [24] & 2020 & Internet of things & CNN and LSTM & $\begin{array}{l}\text { The CNN is used in an IoT micro- } \\
\text { security add-on, while the LSTM is used } \\
\text { by the back-end server }\end{array}$ & N-BaIoT dataset & $\begin{array}{l}\text { F1 score, True Positive } \\
\text { Rate, True Negative Rate, } \\
\text { precision, Accuracy, recall }\end{array}$ \\
\hline Latif et al. [25] & 2020 & Industrial internet of things & $\begin{array}{l}\text { Lightweight random neu- } \\
\text { ral network, }\end{array}$ & $\begin{array}{l}\text { Uses a model with } 1 \text { input layer, } 8 \text { hid- } \\
\text { den layers, and } 1 \text { output layer }\end{array}$ & DS2OS dataset & $\begin{array}{l}\text { Accuracy, precision, recall, } \\
\text { and F1 score }\end{array}$ \\
\hline $\begin{array}{l}\text { Manimurugan } \\
\text { et al. [26] }\end{array}$ & 2020 & Internet of Medical Things & $\begin{array}{l}\text { Deep belief network tech- } \\
\text { nique }\end{array}$ & $\begin{array}{l}\text { Uses the greedy layer-wise scheme to } \\
\text { optimize the deep learning structure }\end{array}$ & CICIDS 2017 dataset & $\begin{array}{l}\text { Accuracy, detection } \\
\text { rate, precision, recall, } \\
\text { F-measure }\end{array}$ \\
\hline $\begin{array}{l}\text { Koroniotis et al. } \\
{[27]}\end{array}$ & 2020 & Internet of things & Deep Neural Network & $\begin{array}{l}\text { Detecting IoT attacks based on three } \\
\text { stages, namely, extracting data, adapt } \\
\text { parameters of deep learning, and iden- } \\
\text { tify the anomalous incidents }\end{array}$ & $\begin{array}{l}\text { Bot-IoT and } \\
\text { UNSW_NB15 datasets }\end{array}$ & $\begin{array}{l}\text { Recall, F-measure, accu- } \\
\text { racy, precision }\end{array}$ \\
\hline Zhou et al. [28] & 2020 & Industry 4.0 & $\begin{array}{l}\text { Variational long short- } \\
\text { term memory (VLSTM) } \\
\text { learning model }\end{array}$ & $\begin{array}{l}\text { Detecting IoT attacks based a en- } \\
\text { coder-decoder neural network }\end{array}$ & UNSW_NB15 dataset & $\begin{array}{l}\text { Accuracy, False alarm rate, } \\
\text { F1, Area under curve }\end{array}$ \\
\hline $\begin{array}{l}\text { NG and Selvaku- } \\
\text { mar [29] }\end{array}$ & 2020 & $\begin{array}{l}\text { Fog computing-enable Inter- } \\
\text { net of things }\end{array}$ & $\begin{array}{l}\text { Convolutional deep learn- } \\
\text { ing technique }\end{array}$ & $\begin{array}{l}\text { The computations are performed in the } \\
\text { fog nodes }\end{array}$ & $\begin{array}{l}\text { UNSW's } \\
\text { dataset }\end{array}$ & $\begin{array}{l}\text { Accuracy, precision, recall, } \\
\text { F-measure }\end{array}$ \\
\hline Khoa et al. [30] & 2020 & IoT industry 4.0 & Deep neural networks & $\begin{array}{l}\text { Uses smart "filters" deployed at the IoT } \\
\text { gateways for detecting network attacks }\end{array}$ & $\begin{array}{l}\text { - KDD, NSL-KDD, and } \\
\text { UNSW } \\
\text { - N-BaIoT dataset }\end{array}$ & - Accuracy \\
\hline $\begin{array}{l}\text { Ferrag and Lean- } \\
\text { dros [14] }\end{array}$ & 2020 & Smart Grids & Recurrent neural networks & $\begin{array}{l}\text { Employs recurrent neural networks } \\
\text { with blockchain for detecting network } \\
\text { attacks }\end{array}$ & $\begin{array}{l}\text { - Bot-IoT dataset } \\
\text { - CICIDS2017 dataset } \\
\text { - Power system dataset }\end{array}$ & $\begin{array}{l}\text { - False alarm rate, detec- } \\
\text { tion rate, accuracy }\end{array}$ \\
\hline Popoola et al. [31] & 2020 & Internet of Things & $\begin{array}{l}\text { Deep bidirectional long } \\
\text { short-term memory }\end{array}$ & $\begin{array}{l}\text { Uses deep bidirectional long short-term } \\
\text { memory to identify the traffic of botnet } \\
\text { attacks from benign traffic in IoT net- } \\
\text { works }\end{array}$ & Bot-IoT dataset & $\begin{array}{l}\text { Matthews Correlation Co- } \\
\text { efficient }\end{array}$ \\
\hline $\begin{array}{l}\text { Al-Hawawreh } \\
\text { et al. [32] }\end{array}$ & 2020 & Internet of Things & Deep learning techniques & $\begin{array}{l}\text { Uses a deep pattern extractor to identify } \\
\text { the attack types of malicious patterns }\end{array}$ & $\begin{array}{l}\text { - TON-IoT dataset } \\
\text { - N-BAIOT dataset }\end{array}$ & $\begin{array}{l}\text { Accuracy, DR, FPR, FNR, } \\
\text { MCC }\end{array}$ \\
\hline Ge et al. [33] & 2021 & Internet of Things & $\begin{array}{l}\text { Customised deep learning } \\
\text { technique }\end{array}$ & $\begin{array}{l}\text { Uses the concepts of deep learning and } \\
\text { transfer learning for cyber security in } \\
\text { IoT networks }\end{array}$ & Bot-IoT dataset & $\begin{array}{l}\text { Accuracy, Recall, Precision, } \\
\text { and F1 score }\end{array}$ \\
\hline Our Work & / & Agriculture 4.0 & $\begin{array}{l}\text { Convolutional neural net- } \\
\text { work, Deep neural net- } \\
\text { work, and Recurrent neu- } \\
\text { ral network }\end{array}$ & $\begin{array}{l}\text { Study the performance of three deep } \\
\text { learning models to identify the traffic } \\
\text { of DDoS attacks from benign traffic in } \\
\text { Agriculture } 4.0\end{array}$ & $\begin{array}{l}\text { - CIC-DDoS2019 } \\
\text { dataset [34] } \\
\text { - TON_IoT dataset [35] }\end{array}$ & $\begin{array}{l}\text { Detection rate (DR), false } \\
\text { alarm rate (FAR), preci- } \\
\text { sion, F-score, recall, True } \\
\text { Negative Rate(TNR), False } \\
\text { Accept Rate (FAR), ROC } \\
\text { Curve, and accuracy }\end{array}$ \\
\hline
\end{tabular}

Diro and Chilamkurti [20] designed a distributed attack detection system based on deep learning for the IoT networks. The authors proposed to deploy this system at the fog computing layer for hosting attack detection systems and training models. Three cyber security datasets are used in the performance evaluation, including, NSL-KDD, ISCX, and KDDCUP99, in which the results show a precision of $71 \%, 98.56 \%$, and $97 \%$, for R2L.U2R attacks, Probe attacks, and DoS attacks, respectively. Muna et al. [21] an anomaly detection system, named ADS, for detecting cyber attacks in the industrial internet of things. The ADS system uses deep learning techniques, in which the unsupervised deep auto-encoder algorithm is used to train network normal patterns of behavior and generate the correct settings. Both NSL-KDD and UNSW-NB15 are used in the evaluation of performance 
which the results of the experiments demonstrate a detection ratio of $99 \%$ and a false positive ratio of $1.8 \%$.

Based on scanning the DNS services in smart city applications of IoT, Vinayakumar et al. [23] presented an instruction detection mechanism against botnet attacks. Specifically, the proposed mechanism uses a two-tier environment for monitoring DNS logs and searching the domain name generated by the domain generation algorithm through deep learning algorithms to minimize false alarm rates. Latif et al. [25] designed an intrusion detection mechanism that uses a lightweight random neural network for detecting cyber threats in the industrial internet of things. Compared to traditional machine learning techniques such as SVM, ANN, and decision tree, the proposed system shows good performance on an open-source dataset called DS2OS. The DS2OS dataset contains seven types of attacks, including, DoS attacks, Scan, Data type probing, Malicious control, Wrong setup, Spying, and Malicious operation. These attacks are not sufficient to prove the performance of an intrusion detection mechanism for identifying cyber threats in the industrial IoTs.

To identify an adversary who tries to insert useless data and detecting phishing and Botnet attack, Parra et al. [24] proposed distributed architecture using two deep learning approaches, namely, a Distributed CNN scheme and LSTM network. The DCNN is used in an IoT micro-security add-on, while the LSTM is used by the back-end server. The N-BaIoT dataset is employed in the evaluation of performance, where outcomes show an accuracy of $98 \%$ and $94.30 \%$ during the training phase and the testing phase, respectively. To detecting IoT malware, Haddad Pajouh et al. [22] designed an intrusion detection mechanism using a recurrent neural network. The proposed mechanism employs three stages; namely, collection data, feature extrication, and deep threat classifier. The results of the experiments demonstrate the highest accuracy of $98.18 \%$ under the 10 -fold cross-validation analysis and efficient compared to conventional machine learning classifiers such as Naive Bayes, K-Nearest Neighbor, Random Forest, and Decision Tree. Koroniotis et al. [27] proposed a network forensics scheme, called PDF, for detecting and monitoring the attack patterns in IoT based networks. The PDF scheme is based on three stages, namely, (1) extracting data, (2) adapt parameters of deep learning, and (3) identify anomalous incidents. The particle swarm optimization algorithm is used in the second stage, whereas the deep neural model is used in the third stage. The experimental results reveal an accuracy of $99.9 \%$ compared to $93.2 \%$ with decision tree and $72.7 \%$ with naïve bayes.

NG and Selvakumar [29] proposed an anomaly detection framework based on vector convolutional deep learning technique. The authors proposed also that the computations are processed at the fog nodes. The experiments conducted on the UNSW Bot-IoT dataset show an accuracy of $99.71 \%, 99.80 \%, 99.92 \%, 77.22 \%$, for DDoS attacks, DoS attacks, Reconnaissance attacks, and Theft attacks, respectively. Therefore, to detect cyber attacks in the Internet-of-Medical-Things (IMoT), Manimurugan et al. [26] introduced an intrusion detection mechanism using the deep belief network technique. The proposed mechanism is evaluated using the CICIDS 2017 dataset which shows an accuracy of $97.71 \%$ and $96.37 \%$ for PortScan attack and infiltration attack, respectively. Popoola et al. [31] developed a hybrid intrusion detection mechanism, called LAE-BLSTM, for the detection of botnets in IoT networks. The LAE-BLSTM mechanism uses deep Bidirectional Long Short-Term Memory (BLSTM) and Long Short-Term Memory Autoencoder (LAE). The LAE is used for the dimensionality reduction of the feature, while the BLSTM is used to identify the traffic of botnet attacks from benign traffic in IoT networks. The Bot-IoT dataset used in the evaluation of performance, which demonstrates that the LAE-BLSTM mechanism reached a data size reduction ratio of $91.89 \%$.

\section{IDS Implementation}

In this section, we propose three deep learning-based IDS models for detection of cyber attacks in Agriculture 4.0, including recurrent neural network-based IDS model, convolutional neural network-based IDS model, and deep neural network-based IDS model. 


\subsection{Network Model}

The considered network model of Agriculture 4.0 is presented in Figure 3, which is based on three layers, namely, (1) Agricultural sensors layer, (2) Fog computing layer, and (3) Cloud computing layer. The agricultural sensors layer consists of various IoT devices and drones applied to monitor agricultural environment data. Actuators are activated in the agricultural sensors layer when the data meet specific conditions. New energy technology and smart grid architecture are placed in the agricultural sensors layer for supplying energy for IoT devices. In each fog node, a deep learning based-intrusion detection system is placed. The IoT data are transmitted directly to the fog computing layer from the agricultural sensors layer for analysis and machine learning algorithms, while Cloud computing nodes provide the storage and end-to-end services. The computations of deep learning based-intrusion detection systems are performed in the fog nodes. We consider that there is a group of attackers that launch DDoS attacks in order to affect the functioning of the network, which can affect food safety, agri-food supply chain efficiency, and agricultural productivity.

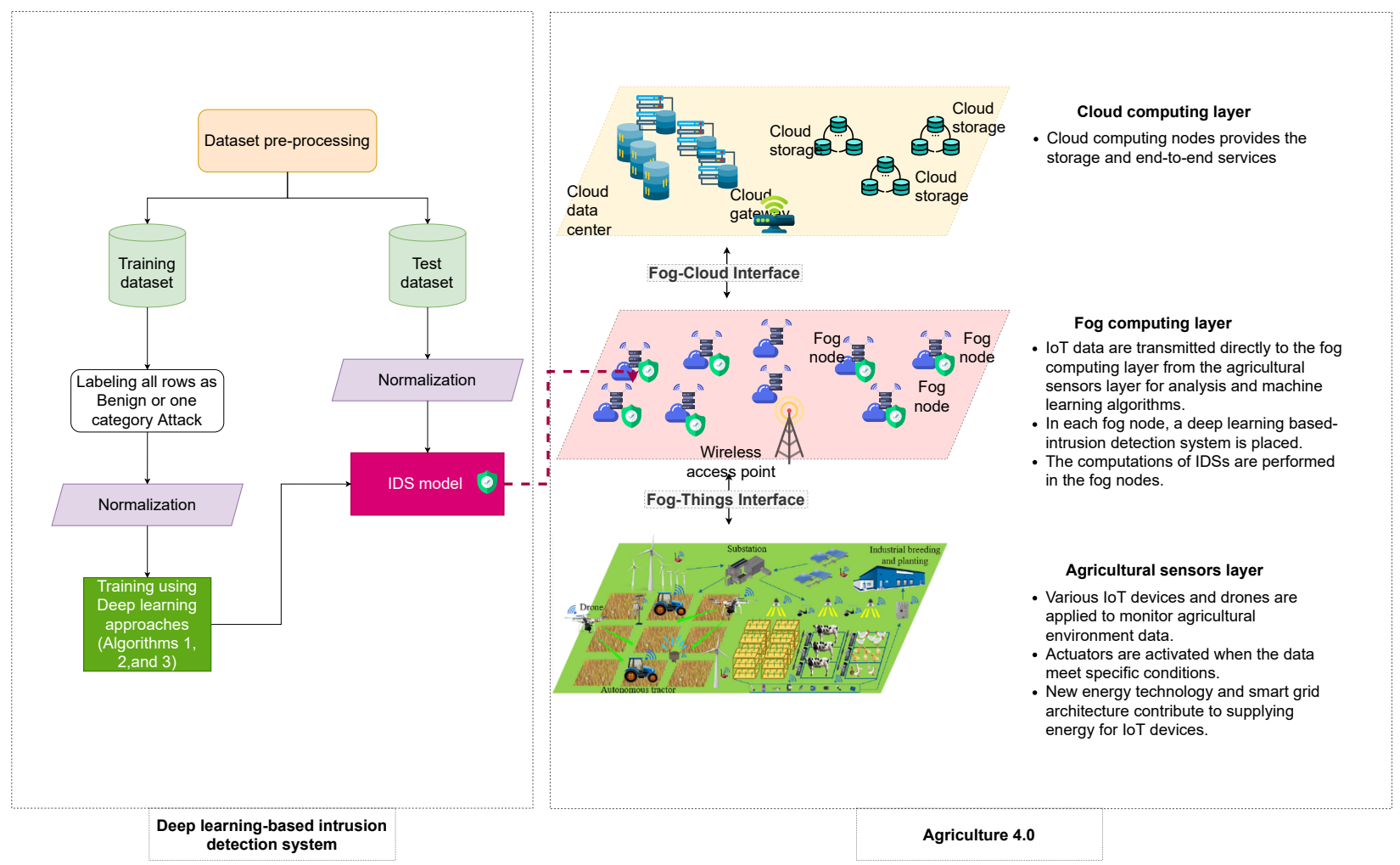

Figure 3. The proposed deep learning-based IDS for DDoS attack in Agriculture 4.0.

\subsection{Rnn-Based Ids}

Recurrent neural networks, or RNN, are a category of neural networks for processing sequential data (i.e., a sequence of values $X^{(1)}, \ldots, X^{(T)}$ ). It allows the previous predictions to be used as inputs, using hidden states. The RNN is based on the multilayer perceptron which is an acyclic neural network structured in layers, namely, an input layer, one or more intermediate layers called hidden layers, and an output layer. The multilayer perceptron is described by the $n$ layers that compose it and which are successive. The layer $L \in[[1, N]]$ of multilayer perceptron is defined by : $T_{-} L=\left(n_{-} L, \sigma_{-} L, a_{-} L\right)$ where $n_{-} L \in \mathbb{N}$ is the number of neurons in the layer $L_{\text {. }} a_{-} L: \mathbb{R}^{n_{-} L_{-1}} \rightarrow \mathbb{R}^{n_{-} L}$ is the affine transformation defined by the vector $b_{-} L \in \mathbb{R}^{n-L}$ and the matrix $W_{-} L \in \mathbb{R}^{n-L-1 \times n_{-} L}$. Note that the $b_{-} L$ is a bias vector, which is an additive set of weights in a neural network that requires no input.

For an input vector sequence $x(t)$ containing the features of an input dataset of attacks with $t \in\left[\left[1, t_{-} f\right]\right]$, we have an output vector sequence $o(t)$ with $t \in\left[\left[1, t_{-} f\right]\right]$ by the initialization of the internal state vectors as follows : 


$$
\text { State_0 }(t)=x(t), \forall t \in\left[\left[1, t_{-} f\right]\right]
$$

and

$$
\text { State } L(0)=x(0), \forall t \in[[1, N]]
$$

Then, for each time step, all the layers of the network are recursively applied to the previous layer's output vector:

$$
\begin{gathered}
g \_L(t)=W \_L \times h \_L-1(t)+V \_L \times h \_L(t-1)+b \_L \\
h \_L(t)=\sigma \_L\left(g \_L(t)\right) \\
o(t)=h \_N(t)
\end{gathered}
$$

To alleviate the vanishing gradient problem, there are two solution, namely, Gated recurrent units (GRUs) and Long short-term memory (LSTM). We use in our algorithm the LSTM which is one of the most popular RNN architectures to date. The LSTM is described as follows [36]:

$$
\begin{aligned}
& \text { Cell_t }=\text { Forget_t } \odot \text { Cell_t }-1+\operatorname{Input} \_t \odot \text { Cẽll_t } \\
& \text { Forget_t }=\sigma\left(W_{-} f h h_{-} t-1+W_{-} f x x_{-} t+b_{-} f\right) \\
& \text { Output_t }=\sigma\left(W \_o h{ }_{-} t-1+W \_o x x \_t+b \_o\right) \\
& \text { Input_t }=\sigma\left(W_{-} i h h_{-} t-1+W_{-} i x x_{-} t+b_{-} i\right) \\
& \text { Cêll_t }=\tanh \left(W \_c h h \_t-1+W \_c x x \_t+b \_c\right) \\
& \text { Hidden_t }=\text { Output_t } \odot \tanh \left(C e l l_{-} t\right)
\end{aligned}
$$

Where Forget_t is the forget gate, Cell_t is the memory cell, Input_t is the input gate, Output_t is the output gate, and Hidden_t is the new hidden state.

The proposed RNN-based IDS architecture for detection of cyber attacks in Agriculture 4.0 is presented in Algorithm 1 which is written in Python language. The description of each functions used in Algorithms 1-3 are presented in Table 2.

Table 2. Functions used in Algorithms 1-3.

\begin{tabular}{ll}
\hline Function & Description \\
\hline model $=$ Sequential () & Create a sequential model incrementally via the add () method. \\
\hline add () & The add () method consists of adding layers. \\
\hline Dropout () & $\begin{array}{l}\text { The dropout is a regularization technique for neural networks and } \\
\text { deep learning models, where randomly selected neurons are ignored } \\
\text { during training. }\end{array}$ \\
\hline Dense () & The dense layer is the regular deeply connected neural network layer. \\
\hline LSTM() & Adding the Long Short-Term Memory layer. \\
\hline return_sequences & $\begin{array}{l}\text { Determines whether to return the last output in the output sequence } \\
\text { or the full sequence. }\end{array}$ \\
\hline input_shape & The shape of our training set. \\
\hline compile( () & Compile the model. \\
\hline model.fit () & Train the model, iterating on the data in batches of $X$ samples. \\
\hline training_loss & Get training loss histories. \\
\hline test_loss & Get test loss histories. \\
\hline plt.show () & Visualize the confusion matrix. \\
\hline
\end{tabular}


Table 2. Cont.

\begin{tabular}{|c|c|}
\hline Function & Description \\
\hline Conv1D() & $\begin{array}{l}\text { The Conv1D consists of creating a convolution kernel that is con- } \\
\text { volved with the layer input. }\end{array}$ \\
\hline Global AveragePooling1D() & Convert each feature map into one value. \\
\hline np.argmax & Create the confusion matrix. \\
\hline$S G D()$ & $\begin{array}{l}\text { Implements the stochastic gradient descent optimizer with a learning } \\
\text { rate and momentum. }\end{array}$ \\
\hline Accuracy & $\begin{array}{l}\text { The number of correct predictions made as a ratio of all predictions } \\
\text { made. }\end{array}$ \\
\hline AreaUnderROCCurve & $\begin{array}{l}\text { A plot of the true positive rate and the false positive rate for a given } \\
\text { set of probability predictions. }\end{array}$ \\
\hline ConfusionMatrix & $\begin{array}{l}\text { The confusion matrix is a handy presentation of the accuracy of a } \\
\text { model with two or more classes. }\end{array}$ \\
\hline classification_report () & $\begin{array}{l}\text { function displays the precision, recall, f1-score and support for each } \\
\text { class. }\end{array}$ \\
\hline $\operatorname{ReLU}$ & The rectified linear activation function. \\
\hline Sigmoid & $\begin{array}{l}\text { The sigmoid activation function that takes any real value as input } \\
\text { and outputs values in the range } 0 \text { to } 1 \text {. }\end{array}$ \\
\hline Tanh & $\begin{array}{l}\text { The hyperbolic tangent activation function that takes any real value } \\
\text { as input and outputs values in the range }-1 \text { to } 1 \text {. }\end{array}$ \\
\hline
\end{tabular}

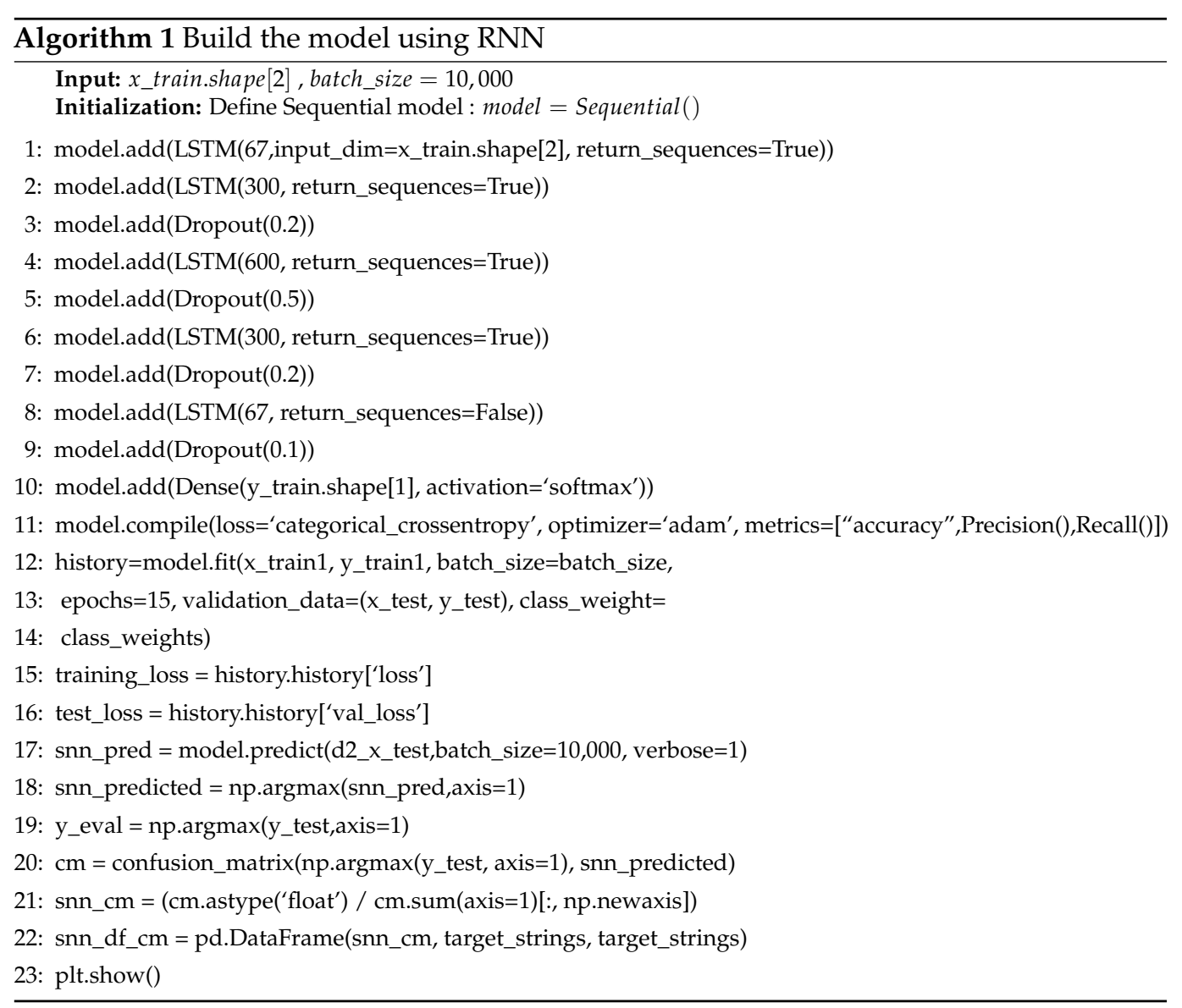



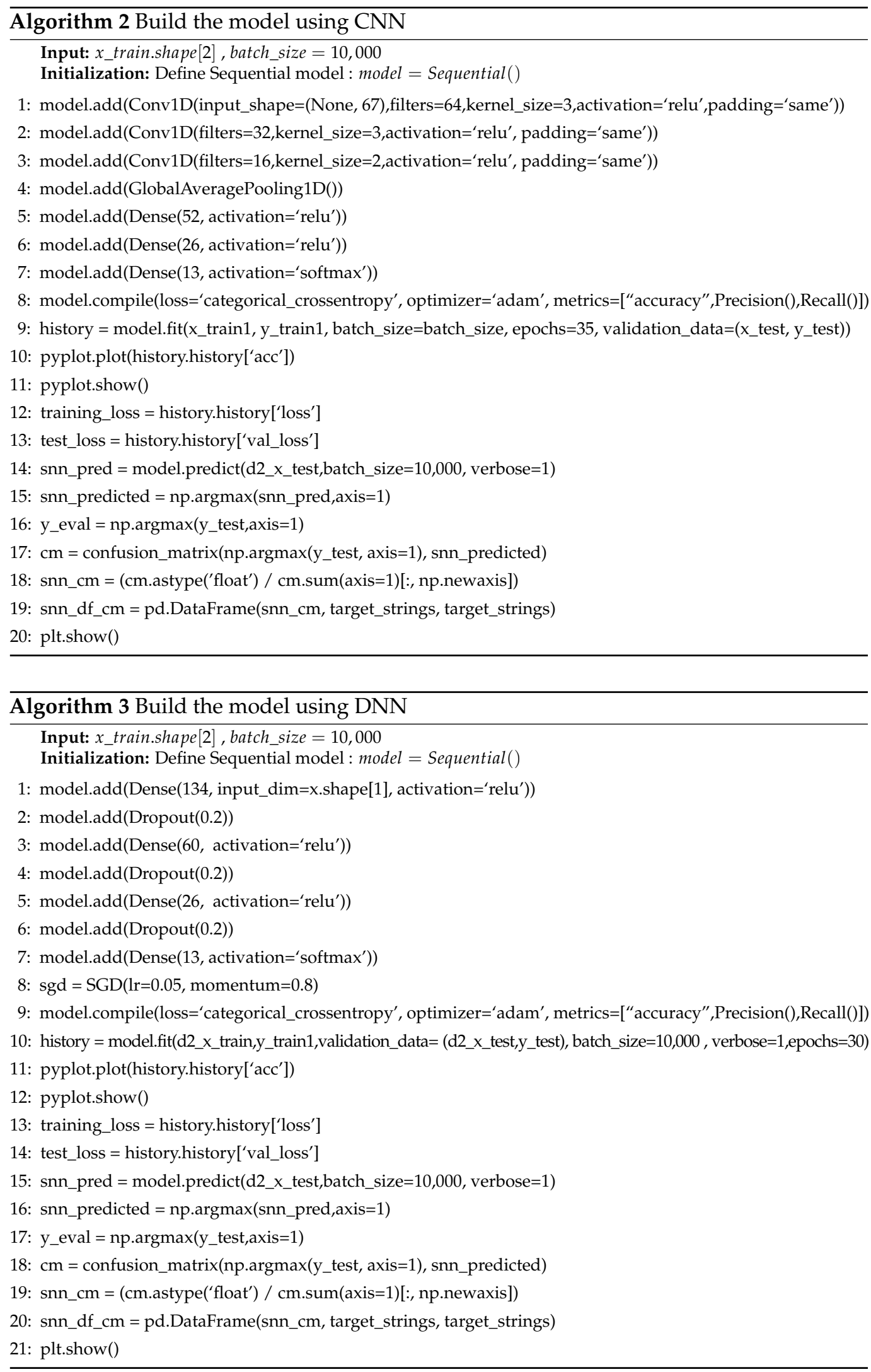

\subsection{Cnn-Based Ids}

Convolutional networks, also known as convolutional neural networks, or CNNs, represent a dedicated class of neural network for data processing with a familiar network structure. The name "convolutional neural network" means that the network uses a 
mathematical operation called convolution [37]. Therefore, a convolutional neural network structure is composed of a set of processing layers, as follows:

- The convolution layer (CONV) that manages the data from a receiver cell. There are three hyperparameters to dimension the volume of the convolution layer: the depth, stride, and zero-padding. The formula for calculating the number of neurons in the output volume $W \_o$ is described as follows:

$$
W \_o=\frac{W \_i-S+2 M}{P}+1
$$

where $W \_i$ is the size of the input volume, $S$ is the kernel field size of the convolutional layer neurons, $M$ is the amount of zero padding, and $P$ is the stride.

- The pooling layer (POOL), which enables to reduce the size of the intermediate image by compressing the information and operates on each feature map independently.

- The correction layer (Rectified Linear Unit, ReLU), which is often referred to as the "ReLU" in reference to the activation function. The ReLU applies the non-saturating activation function, which is described as follows:

$$
\text { function }(x)=\max (0, x)
$$

Note that there are other functions that can be used to increase nonlinearity, such as the sigmoid function, which is described as follows:

$$
S(x)=\frac{e^{x}}{e^{x}+1}
$$

- The "fully connected" (FC) layer is a perceptron-type layer.

- The loss layer as the final layer of a neural network. Different loss functions can be used such as Euclidean loss, Softmax loss, and Sigmoid cross-entropy loss.

The proposed CNN-based IDS architecture for detection of cyber attacks in Agriculture 4.0 is presented in Algorithm 2 which is written in Python language.

\subsection{Dnn-Based Ids}

A deep neural network (DNN) is an artificial neural network (ANN) with more layers intermediate between input and output layers. The DNN consist of five-part: neurons, weights, synapses, biases, and functions. The inputs $\left(X=x \_1, \ldots, x \_n\right)$ are linked with weights $\left(W=w_{-} \_1 \ldots w_{-} n\right)$. The full-fledged deep learning system is based on multilayer perception (defined in RNN-based IDS) with the application of various activation functions that produce real values, rather than Boolean values as in the classical perceptron. To help to adjust the input weights and minimize the "loss", the backpropagation algorithm is applied to performs iterative backward passes. The proposed DNN-based IDS architecture for detection of cyber attacks in Agriculture 4.0 is presented in Algorithm 3 which is written in Python language based on the following packages Pandas (https://pandas. pydata.org/pandas-docs/stable/ (accessed on 1 April 2021 )), NumPy (https:/ /numpy. org (accessed on 1 April 2021)), SciPy (https://scipy.org (accessed on 1 April 2021)), TensorFlow (https://tensorflow.org/ (accessed on 1 April 2021)), and Keras (https:// keras.io/ (accessed on 1 April 2021)).

\section{Performance Evaluation}

Agriculture 4.0 consists of integrating advanced technologies into existing farm operations to improve the quality and productivity of agricultural products. These advanced technologies include IoT devices, 5G communications, Drones, Fog/Edge computing, Cloud Computing, Artificial Intelligence, Network Function Virtualization, and Software-Defined Networking. Based on these technologies, we used and selected the most recent data sets that contain DDoS attack scenarios against these technologies used by Agriculture 4.0. 
Specifically, we used the following two new real traffic datasets, namely, CIC-DDoS2019 dataset [34] and TON_IoT dataset [35]. They are chosen for three reasons: (1) because they were built for a TCP/IP communication stack, (2) contain DDoS attacks, and (3) represent the nature of Agriculture 4.0 (in particular its IoT and IIoT sensors and cloud-edge traffic). The TON_IoT dataset was designed based on interacting network elements and IoT/IIoT systems with the three layers of Edge, Fog, and Cloud to simulate a real-world execution of current production IoT/IIoT networks. The NSX-VMware platform was utilized as Software-Defined Network (SDN) and Network Function Virtualisation (NFV) technologies to facilitate the management of the interaction between these three layers. The experiment is conducted on Google Colaboratory (https: / / colab.research.google.com (accessed on 1 April 2021 )) using python 3 with the Graphics Processing Unit (GPU) and TensorFlow.

The details of the IDS experiment methodology are shown in Figure 4 and an overview of pre-processing of CIC-DDoS2019 dataset [34] and TON_IoT dataset [35] with the Deep learning-based IDS deployment is presented in Figure 5. More precisely, the approach is composed of four steps: (1) datasets step, (2) pre-processing step, (3) training step, and (4) testing step. The hyperparameters employed in deep learning strategies are shown in Table 3.

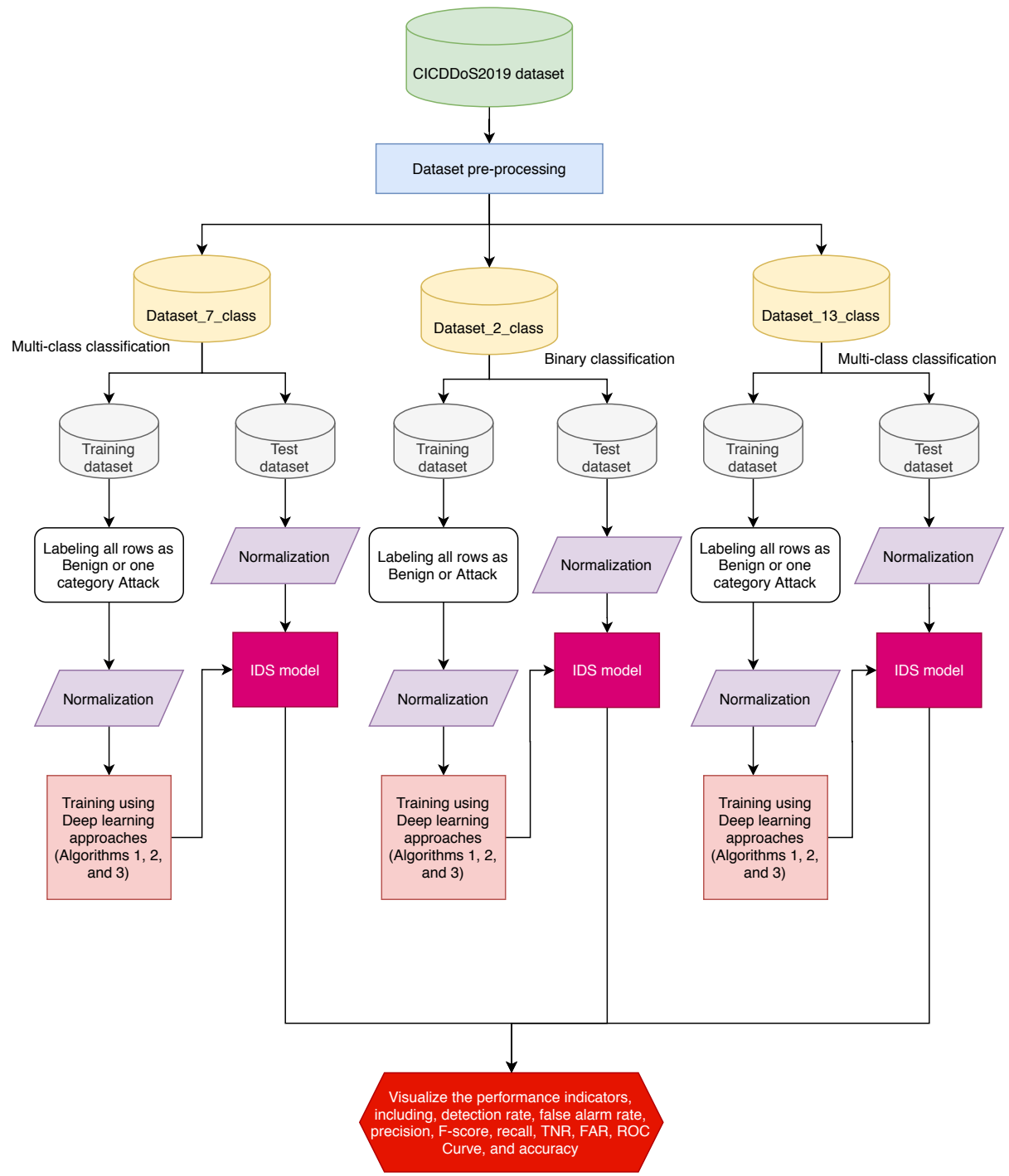

Figure 4. Flowchart of the cyber security intrusion detection methodology. 


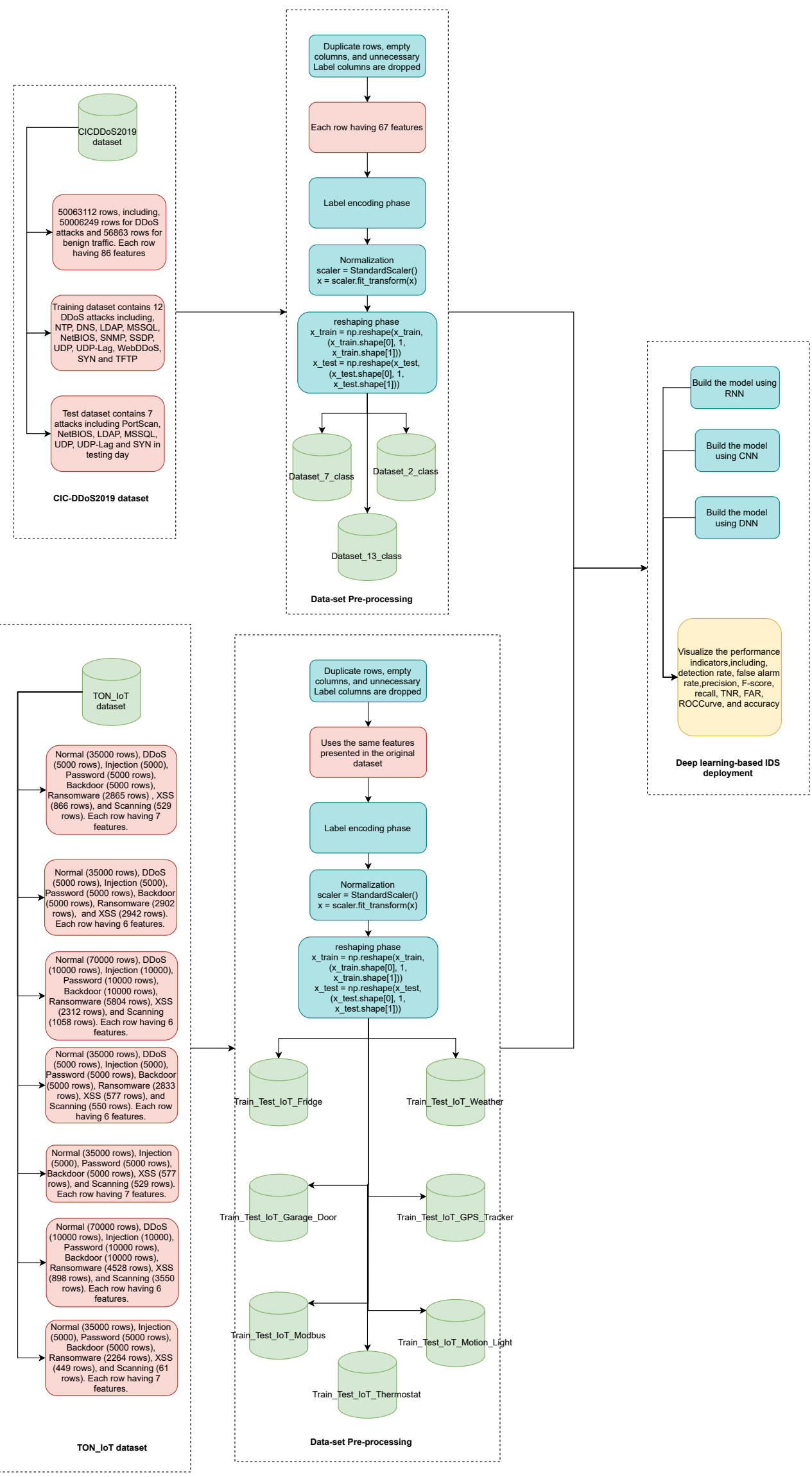

Figure 5. Overview of pre-processing of CIC-DDoS2019 dataset [34] and TON_IoT dataset [35] with Deep learning-based IDS deployment. 
Table 3. The hyperparameters employed in deep learning approaches.

\begin{tabular}{cc}
\hline Hyperparameter & Value \\
\hline Activation function & Sigmoid \\
\hline Classification function & SoftMax \\
\hline Batch size & 10,000 \\
\hline Hidden nodes (HN) & $15-100$ \\
\hline Number of epoch & 100 \\
\hline Learning rate (LR) & $0.01-0.5$ \\
\hline
\end{tabular}

\subsection{Pre-Processing of the Cic-Ddos2019 Dataset}

The CIC-DDoS2019 dataset [34] includes 50,063,112 records, including, 50,006,249 rows for DDoS attacks and 56,863 rows for benign traffic. Each row having 86 features. The statistics for attacks in training and testing for the dataset are summarized in Table 4. The training dataset contains 12 DDoS attacks including, Network Time Protocol (NTP), Domain Name System (DNS), Lightweight Directory Access Protocol (LDAP), Microsoft SQL Server (MSSQL), NETwork Basic Input Output System (NetBIOS), Simple Network Management Protocol (SNMP), Simple Service Discovery Protocol (SSDP), User Datagram Protocol (UDP), UDP-Lag, WebDDoS, SYN and TFTP, while the test dataset contains seven attacks, namely, MSSQL, NetBIOS, PortScan, LDAP, UDP, UDP-Lag and SYN in testing day.

- NTP-based attack: is a DDoS attack based on a reflection where an attacker uses Network Time Protocol (NTP) server functionality to flood a specific client-server or other networks with an increased quantity of UDP data traffic. This attack can make the destination and its network infrastructure unavailable to normal traffic.

- DNS-based attack: is a DDoS attack based on a reflection where an attacker uses a Botnet to create a large number of resolution requests to a targeted IP address.

- LDAP-based attack: is a DDoS attack based on a reflection where an attacker sends requests to a publicly available vulnerable LDAP server to generate large responses (amplified), reflected to a target server.

- MSSQL-based attack: is a DDoS attack based on a reflection where an attacker exploits the Microsoft SQL Server Resolution Protocol (MC-SQLR) by executing scripted requests using a forged IP address in order to appear as coming from the target server.

- NetBIOS-based attack: is a DDoS attack based on a reflection where an attacker sends spoofed "Name Release" or "Name Conflict" messages to a victim machine in order to refuse all NetBIOS network traffic.

- SNMP-based attack: This attack is a volumetric DDoS threat that uses the Simple Network Management Protocol (SNMP) to generate attack volumes of hundreds of gigabits per second in order to clog the target's network pipes.

- SSDP-based attack: is a DDoS attack based on a reflection where an attacker sends an amplified amount of traffic to a targeted victim using Universal Plug and Play (UPnP) networking protocols.

- UDP-Lag-based attack: This attack aims to slow down/interrupt the targeted host with IP packets containing UDP datagrams.

- WebDDoS-based attack: This threat takes advantage of legitimate HTTP GET or POST queries to compromise a Web server or application.

- $\quad$ SYN-based attack: This attack exploits the normal TCP three-way handshake (i.e., sending SYN (synchronize), sending SYN-ACK (synchronize-acknowledge), and responds with an ACK (acknowledge)) to use resources on the targeted network server and make it unresponsive.

- $\quad$ TFTP-based attack: This attack exploits the Trivial File Transfer Protocol (TFTP) by employing TFTP servers connected to the internet. Specifically, an attacker performs 
a request by default for a file, and the victim TFTP server sends the data back to the requesting target host.

- PortScan-based attack: This attack performs a network security audit by conducting port scanning on a specific machine or on an entire network. The scanning is done using queries to determine which services are running on a remote host.

Table 4. Attack types in CICDDoS2019 dataset.

\begin{tabular}{cc}
\hline Attack Type & Flow Count \\
\hline Benign & 56,863 \\
\hline DDoS_DNS & $5,071,011$ \\
\hline DDoS_LDAP & $2,179,930$ \\
\hline DDoS_MSSQL & $4,522,492$ \\
\hline DDoS_NetBIOS & $4,093,279$ \\
\hline DDoS_NTP & $1,202,642$ \\
\hline DDoS_SNMP & $5,159,870$ \\
\hline DDoS_SSDP & $2,610,611$ \\
\hline DDoS_SYN & $1,582,289$ \\
\hline DDoS_TFTP & $20,082,580$ \\
\hline DDoS_UDP & $3,134,645$ \\
\hline DDoS_UDP-Lag & 366,461 \\
\hline DDoS_WebDDoS & 439 \\
\hline
\end{tabular}

To analyze the efficiency of machine learning and deep learning strategies in terms of binary classification (i.e., Classification tasks with two classes) and multi-class classification (i.e., Classification tasks with more than two classes), We create three different datasets, named Dataset_2_class, Dataset_7_class, and Dataset_13_class. The statistics for attacks in training and testing for each dataset are summarized in Tables $5-7$, respectively.

Table 5. Attack types in Dataset_2_class.

\begin{tabular}{ccc}
\hline Category & Training & Test \\
\hline Benign & 56,101 & 17,146 \\
\hline Attack & 997,054 & 314,716 \\
\hline
\end{tabular}

Table 6. Attack types in Dataset_7_class.

\begin{tabular}{cccc}
\hline Category & Type of Attack & Training & Test \\
\hline \multirow{3}{*}{ Reflection-based attacks } & DrDoS_NetBIOS & 619,700 & 136,729 \\
\cline { 2 - 4 } & DrDoS_MSSQL & 619,446 & 157,076 \\
\cline { 2 - 4 } & DrDoS_LDAP & 619,251 & 150,701 \\
\hline \multirow{2}{*}{\begin{tabular}{c} 
Exploitation-based attacks \\
\cline { 2 - 4 }
\end{tabular}} & DrDoS_UDP & 618,696 & 150,706 \\
\cline { 2 - 4 } & UDP-lag & 183,662 & 1873 \\
\hline $\begin{array}{c}\text { Exploitation/Reflection } \\
\text {-based attacks }\end{array}$ & Syn & 790,662 & 150,416 \\
\hline Benign & Others DoS attacks & 938,733 & 28,127 \\
\hline & Benign & 56,101 & 17,146 \\
\hline
\end{tabular}


Table 7. Attack types in Dataset_13_class.

\begin{tabular}{|c|c|c|c|}
\hline Category & Type of Attack & Flow Count & Training/ Test \\
\hline BENIGN & BENIGN & 56,101 & \multirow{13}{*}{$\begin{array}{c}\text { Splitiing the data } \\
\text { between train / test } \\
\text { x_train, } x \text { _test, } y \_ \text {train, } y \_ \text {test } \\
=\text { train_test_split }(x, y, \\
\text { test_size }=0.25, \text { stratify }=y)\end{array}$} \\
\hline \multirow{9}{*}{$\begin{array}{c}\text { Reflection } \\
\text {-based attacks }\end{array}$} & DrDoS_LDAP & 99,943 & \\
\hline & DrDoS_SSDP & 98,576 & \\
\hline & DrDoS_DNS & 96,567 & \\
\hline & DrDoS_MSSQL & 95,700 & \\
\hline & DrDoS_NetBIOS & 93,560 & \\
\hline & DrDoS_SNMP & 91,578 & \\
\hline & DrDoS_NTP & 76,457 & \\
\hline & TFTP & 72,116 & \\
\hline & WebDDoS & 439 & \\
\hline \multirow{3}{*}{$\begin{array}{c}\text { Exploitation } \\
\text {-based attacks }\end{array}$} & DrDoS_UDP & 97932 & \\
\hline & Syn & 99983 & \\
\hline & UDP-lag & 74203 & \\
\hline
\end{tabular}

\subsection{Pre-Processing of the Ton_iot Dataset}

The TON_IoT dataset [35] is a new testbed for an IIoT network that contains three types of data, namely, network data, operating systems data, and telemetry data [38]. The telemetry datasets of IoT and IIoT sensors are presented in 7 files as presented in Table 8. The contents of these files are described as following:

- $\quad$ File 1 "Train_Test_IoT_Weather": It contains Normal (35,000 rows), DDoS (5000 rows), Injection (5000), Password (5000 rows), Backdoor (5000 rows), Ransomware (2865 rows), XSS (866 rows), and Scanning (529 rows). The file presents the IoT data of temperature measurements, pressure readings, and humidity readings of a weather sensor linked to the network.

- File 2 "Train_Test_IoT_Fridge": It contains Normal (35,000 rows), DDoS (5000 rows), Injection (5000), Password (5000 rows), Backdoor (5000 rows), Ransomware (2902 rows), and XSS (2942 rows). The file presents the IoT data of temperature measurements and temperature conditions of a fridge sensor linked to the network.

- File 3 "Train_Test_IoT_Garage_Door": It contains Normal (70,000 rows), DDoS (10,000 rows), Injection (10,000), Password (10,000 rows), Backdoor (10,000 rows), Ransomware (5804 rows), XSS (2312 rows), and Scanning (1058 rows). The file presents the IoT data of a door sensor linked to the network where the door is closed or open.

- File 4 "Train_Test_IoT_GPS_Tracker": It contains Normal (35,000 rows), DDoS (5000 rows), Injection (5000), Password (5000 rows), Backdoor (5000 rows), Ransomware (2833 rows), XSS (577 rows), and Scanning ( 550 rows). The file presents the IoT data of latitude value and longitude value of GPS tracker sensor linked to the network.

- File 5 "Train_Test_IoT_Modbus": It contains Normal (35,000 rows), Injection (5000), Password (5000 rows), Backdoor (5000 rows), XSS (577 rows), and Scanning (529 rows). The file presents the IoT data of Modbus function code that is responsible for reading an input register.

- File 6 "Train_Test_IoT_Motion_Light": It contains Normal (70,000 rows), DDoS (10,000 rows), Injection (10,000), Password (10,000 rows), Backdoor (10,000 rows), Ransomware (4528 rows), XSS ( 898 rows), and Scanning (3550 rows). The file presents the IoT data of a light sensor that is either on or off.

- $\quad$ File 7 "Train_Test_IoT_Thermostat": It contains Normal (35,000 rows), Injection (5000), Password (5000 rows), Backdoor (5000 rows), Ransomware (2264 rows), XSS 
(449 rows), and Scanning (61 rows). The file presents the IoT data of the current temperature reading of a thermostat sensor connected with the network.

Table 8. Attack types in TON_IoT dataset.

\begin{tabular}{|c|c|c|}
\hline TON_IoT Dataset & Attack Type & Flow Count \\
\hline \multirow{8}{*}{ Train_Test_IoT_Weather } & Normal & 35,000 \\
\hline & DDoS & 5000 \\
\hline & Injection & 5000 \\
\hline & Password & 5000 \\
\hline & Backdoor & 5000 \\
\hline & Ransomware & 2865 \\
\hline & XSS & 866 \\
\hline & Scanning & 529 \\
\hline \multirow{7}{*}{ Train_Test_IoT_Fridge } & Normal & 35,000 \\
\hline & DDoS & 5000 \\
\hline & Injection & 5000 \\
\hline & Password & 5000 \\
\hline & Backdoor & 5000 \\
\hline & Ransomware & 5000 \\
\hline & XSS & 2942 \\
\hline \multirow{8}{*}{ Train_Test_IoT_Garage_Door } & Normal & 70,000 \\
\hline & DDoS & 10,000 \\
\hline & Injection & 10,000 \\
\hline & Password & 10,000 \\
\hline & Backdoor & 100,000 \\
\hline & Ransomware & 5804 \\
\hline & XSS & 2312 \\
\hline & Scanning & 1058 \\
\hline \multirow{8}{*}{ Train_Test_IoT_GPS_Tracker } & Normal & 35,000 \\
\hline & DDoS & 5000 \\
\hline & Injection & 5000 \\
\hline & Password & 5000 \\
\hline & Backdoor & 5000 \\
\hline & Ransomware & 2833 \\
\hline & XSS & 577 \\
\hline & Scanning & 550 \\
\hline
\end{tabular}


Table 8. Cont.

\begin{tabular}{|c|c|c|}
\hline TON_IoT Dataset & Attack Type & Flow Count \\
\hline \multirow{6}{*}{ Train_Test_IoT_Modbus } & Normal & 35,000 \\
\hline & Injection & 5000 \\
\hline & Password & 5000 \\
\hline & Backdoor & 5000 \\
\hline & XSS & 577 \\
\hline & Scanning & 529 \\
\hline \multirow{8}{*}{ Train_Test_IoT_Motion_Light } & Normal & 70,000 \\
\hline & DDoS & 10,000 \\
\hline & Injection & 10,000 \\
\hline & Password & 10,000 \\
\hline & Backdoor & 10,000 \\
\hline & Ransomware & 4528 \\
\hline & XSS & 898 \\
\hline & Scanning & 3550 \\
\hline \multirow{7}{*}{ Train_Test_IoT_Thermostat } & Normal & 35,000 \\
\hline & Injection & 5000 \\
\hline & Password & 5000 \\
\hline & Backdoor & 5000 \\
\hline & Ransomware & 2264 \\
\hline & XSS & 449 \\
\hline & Scanning & 61 \\
\hline
\end{tabular}

\subsection{Performance Metrics}

The performance metrics chosen to evaluate machine learning and deep learning strategies is very important. In our study, we focus on the following important performance metrics: detection rate (DR), false alarm rate (FAR), precision, F-score, recall, TNR, FAR, ROC Curve, and accuracy. Table 9 illustrates four possibilities for both correct and erroneous classification.

$$
\begin{aligned}
& T N R \_B E N I G N=\frac{T N \_B E N I G N}{T N \_B E N I G N+F P \_B E N I G N} \\
& F A R=\frac{F P \_B E N I G N}{T N \_B E N I G N+F P \_B E N I G N} \\
& \text { Precision }=\frac{T P \_ \text {Attack }}{\text { TP_Attack } * \text { FP_BENIGN }} \\
& \text { Recall }=\frac{T P \_ \text {Attack }}{T P \_ \text {Attack } * \text { FN_Attack }} \\
& D R \_ \text {Attack }=\frac{T P \_ \text {Attack }}{T P \_ \text {Attack }+ \text { FN_Attack }} \\
& F-\text { score }=2 * \frac{(\text { Precision } * \text { Recall })}{(\text { Precision }+ \text { Recall })} \\
& \text { Accuracy }=\frac{T P \_ \text {Attack }+ \text { TN_BENIGN }}{T P \_ \text {Attack }+ \text { FN_Attack }+T N \_B E N I G N+F P \_B E N I G N}
\end{aligned}
$$




$$
D R \_ \text {Overall }=\frac{\sum T P \_E a c h-\text { Attack }- \text { Type }}{\sum T P \_E a c h-\text { Attack }- \text { Type }+\sum F N_{-E a c h}-\text { Attack }- \text { Type }}
$$

where $T P, T N, F P$, and $F N$ denote true positive, true negative, false positive, and false negative, respectively. The False Positive (FP) indicates the benign data that is incorrectly classified as an attack, while the True Negative (TN) indicates the benign data that is correctly classified as benign. The True Positive (TP) indicates the attack data that is correctly classified as an attack. The False Negative (FN) indicates the attack data that is incorrectly classified as benign.

Table 9. Confusion matrix.

\begin{tabular}{llcc}
\hline \multirow{2}{*}{ Class } & \multicolumn{2}{c}{ Predicted Class } \\
\cline { 3 - 4 } & & Negative Class & Positive Class \\
\cline { 2 - 4 } & Negative class & True negative $(\mathrm{TN})$ & False positive (FP) \\
\cline { 2 - 4 } & Positive class & False negative (FN) & True positive (TP) \\
\hline
\end{tabular}

\subsection{Results}

The performance of deep learning strategies relative to other machine learning strategies (i.e., DT: Decision Tree, RF: Random forests, NB: Naive Bayes, LR: Logistic Regression) in terms of Precision, Recall, and F-score are shown in Figure 6. In term of Precision, the deep learning techniques give good results in comparison to other machine learning strategies, namely, decision tree, random forests, naive bayes, and logistic regression, and the convolutional neural network model provides the higher ratio with $91 \%$. Both in terms of Recall and F-score, deep learning techniques give good results in comparison to other machine learning strategies, in which the convolutional neural network model provides the higher ratio with $90 \%$ and $89 \%$, respectively. The results show that deep learning techniques can provide better performance in cyber security intrusion detection for Agriculture 4.0.

The performance experimental results of deep learning techniques in term of Precision under binary classification and multiclass classification are shown in Figure 7 . The results show that deep learning techniques give a higher positive prediction in terms of binary classification compared to multiclass classification. Specifically, the convolutional neural network model achieves a precision of $99 \%$ in binary classification compared to $90 \%$ in multiclass classification. Therefore, the performance experimental results of deep learning techniques in term of Recall with binary classification compared to multiclass classification are shown in Figure 8. The results show that deep learning techniques give a higher positive prediction in terms of binary classification compared to multiclass classification. Specifically, the deep neural network model achieves a recall of $99 \%$ in binary classification compared to $83 \%$ and $62 \%$ in multiclass classification. In addition, the recurrent neural network model achieves an F-score of $99 \%$ in binary classification compared to $88 \%$ and $56 \%$ in multiclass classification. 


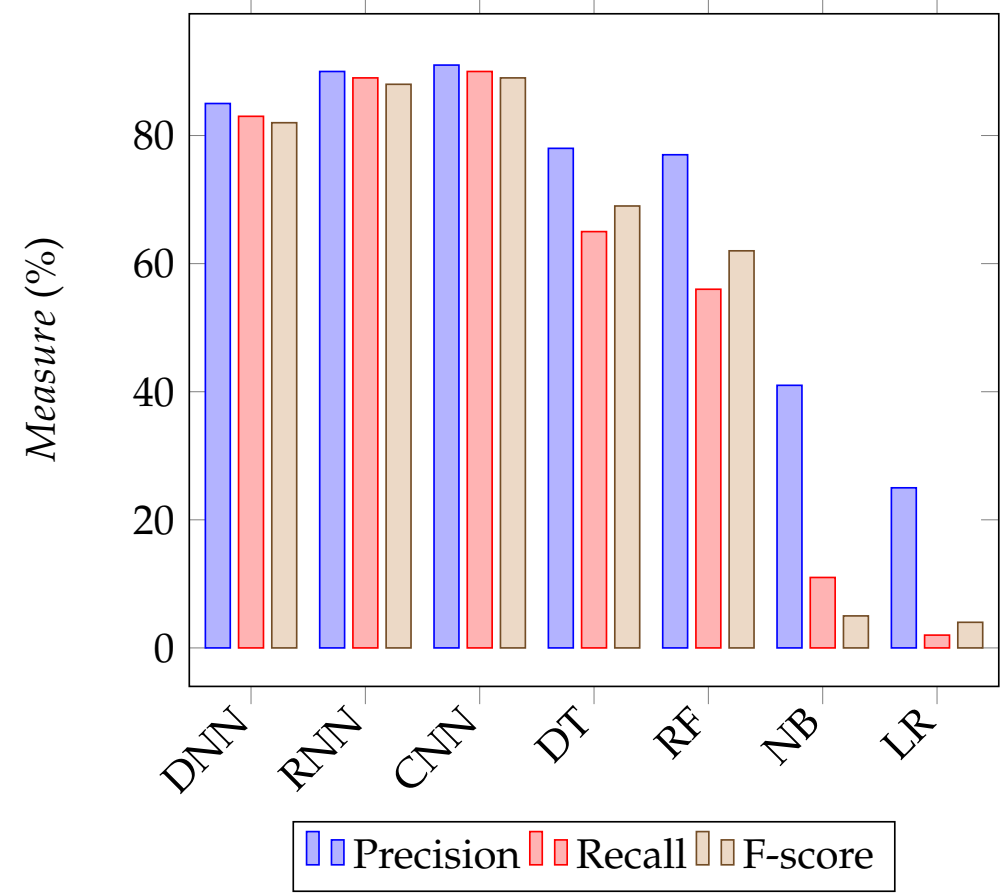

Figure 6. The performance experimental results of deep learning approaches relative to other machine learning strategies in terms of Precision, Recall, and F-score.

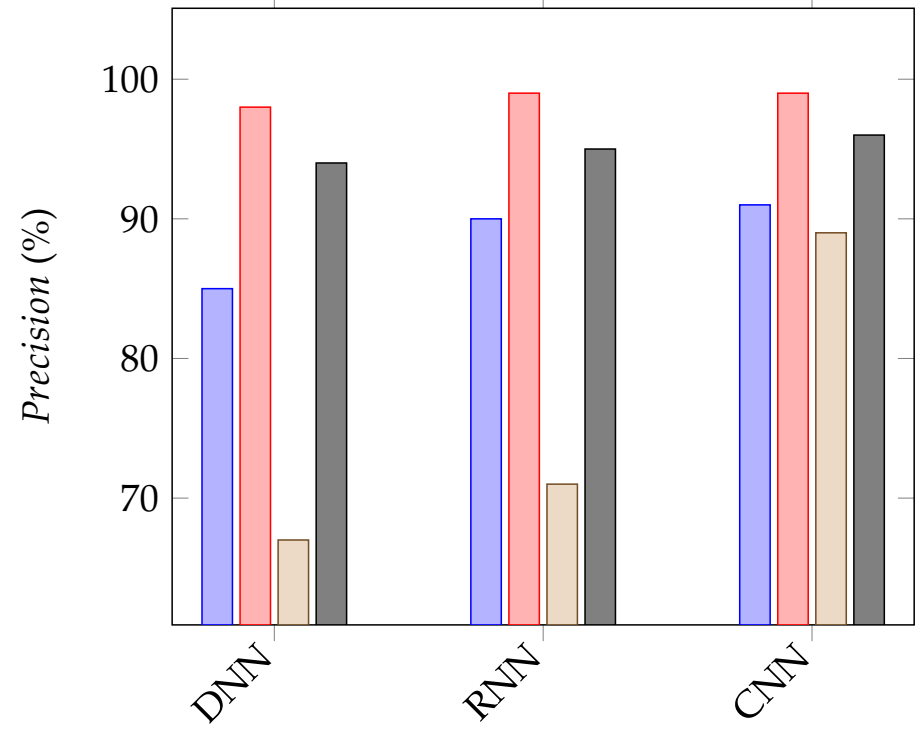

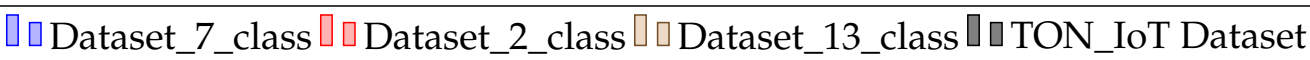

Figure 7. The performance experimental results of deep learning approaches in term of Precision with binary classification and multiclass classification. 


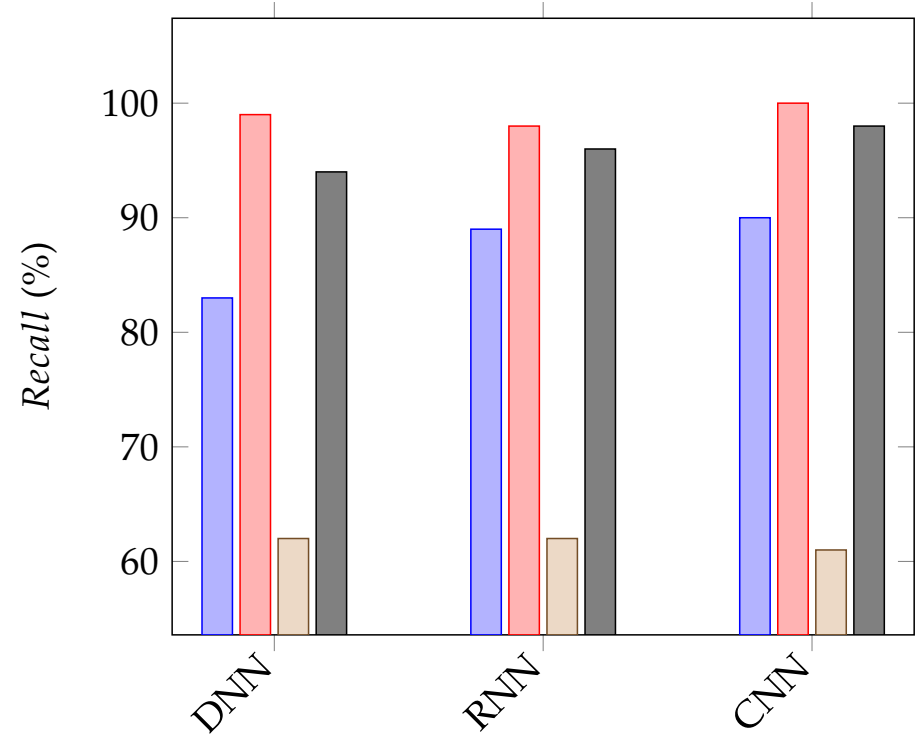

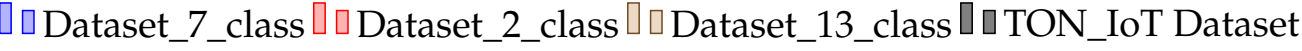

Figure 8. The performance experimental results of deep learning approaches in term of Recall with binary classification and multiclass classification.

Table 10 presents the performance of deep learning approaches relative to benign and various types of attacks in Dataset_7_class (i.e., multi-class classification). We can observe that the convolutional neural network model provides the higher true negative rate with 99\% and the highest detection rate for two attacks type, namely, DrDoS_LDAP and Syn. The deep neural network model gives the higher detection ratio for two attack types, namely, DrDoS_MSSQL and Syn. The recurrent neural network model gives the higher detection ratio for four attack types, namely, DrDoS_LDAP, DrDoS_NetBIOS, DrDoS_UDP, and Syn. In addition, we observe a low detection of UDP-lag attack and this is due to the low learning rate of this attack.

Table 10. The performance experimental results of deep learning approaches relative to benign and various types of attacks in Dataset_7_class (Multi-class classification ).

\begin{tabular}{cccc}
\hline & DNN & RNN & CNN \\
\hline TNR (BENIGN) & $95 \%$ & $98 \%$ & $99 \%$ \\
\hline DrDoS_LDAP & $96 \%$ & $98 \%$ & $97 \%$ \\
\hline DrDoS_MSSQL & $96 \%$ & $94 \%$ & $95 \%$ \\
\hline DrDoS_NetBIOS & $69 \%$ & $99 \%$ & $94 \%$ \\
\hline DrDoS_UDP & $60 \%$ & $71 \%$ & $71 \%$ \\
\hline Syn & $100 \%$ & $100 \%$ & $100 \%$ \\
\hline UDP-lag & $0 \%$ & $0 \%$ & $0 \%$ \\
\hline
\end{tabular}

Table 11 presents the performance of deep learning approaches relative to normal and various types of attacks in TON_IoT dataset (i.e., multi-class classification). We can observe that all three deep learning techniques provide a higher true negative rate. The recurrent neural network model gives a higher detection ratio for three attack types, namely, Injection, Password, and Scanning. The convolutional neural network gives the higher detection ratio for five attacks, namely, DDoS, Backdoor, Ransomware, XSS, and Scanning. 
Table 11. The performance experimental results of deep learning approaches relative to normal and various types of attacks in TON_IoT dataset (Multi-class classification).

\begin{tabular}{cccc}
\hline & DNN & RNN & CNN \\
\hline Normal & $93 \%$ & $97 \%$ & $96 \%$ \\
\hline DDoS & $94 \%$ & $95 \%$ & $98 \%$ \\
\hline Injection & $92 \%$ & $97 \%$ & $94 \%$ \\
\hline Password & $91 \%$ & $97 \%$ & $93 \%$ \\
\hline Backdoor & $93 \%$ & $95 \%$ & $96 \%$ \\
\hline Ransomware & $94 \%$ & $96 \%$ & $97 \%$ \\
\hline XSS & $94 \%$ & $96 \%$ & $97 \%$ \\
\hline Scanning & $94 \%$ & $97 \%$ & $97 \%$ \\
\hline
\end{tabular}

Table 12 presents the performance of deep learning approaches relative to benign and various types of attacks in Dataset_13_class (i.e., multi-class classification). We can observe that all three deep learning techniques provide the higher true negative rate. The deep neural network model gives the higher detection ratio for five attack types, namely, DrDoS_DNS, DrDoS_NTP, DrDoS_SSDP, TFTP, and UDP-lag. The recurrent neural network model gives the higher detection ratio for four attack types, namely, DrDoS_MSSQL, DrDoS_NTP, DrDoS_NetBIOS, and DrDoS_UDP. The convolutional neural network gives the higher detection ratio for Syn attack with $65 \%$. Therefore, we observe a low detection of WebDDoS attack and this is due to the low learning rate of this attack. For binary classification, we can be seen that that all three deep learning techniques provide the higher true negative rate with $99 \%$ and the highest detection rate with $100 \%$, as presented in Table 13.

Table 12. The performance experimental results of deep learning approaches relative to benign and various types of attacks in Dataset_13_class (Multi-class classification).

\begin{tabular}{cccc}
\hline & DNN & RNN & CNN \\
\hline TNR (BENIGN) & $100 \%$ & $100 \%$ & $100 \%$ \\
\hline DrDoS_DNS & $61 \%$ & $56 \%$ & $58 \%$ \\
\hline DrDoS_LDAP & $47 \%$ & $47 \%$ & $47 \%$ \\
\hline DrDoS_SNMP & $67 \%$ & $67 \%$ & $67 \%$ \\
\hline DrDoS_SSDP & $61 \%$ & $58 \%$ & $52 \%$ \\
\hline DrDoS_UDP & $47 \%$ & $48 \%$ & $46 \%$ \\
\hline DrDoS_NetBIOS & $93 \%$ & $97 \%$ & $73 \%$ \\
\hline DrDoS_MSSQL & $55 \%$ & $56 \%$ & $55 \%$ \\
\hline Syn & $64 \%$ & $64 \%$ & $65 \%$ \\
\hline TFTP & $100 \%$ & $99 \%$ & $94 \%$ \\
\hline DrDoS_NTP & $91 \%$ & $91 \%$ & $90 \%$ \\
\hline WebDDoS & $23 \%$ & $24 \%$ & $20 \%$ \\
\hline UDP-lag & $99 \%$ & $98 \%$ & $97 \%$ \\
\hline
\end{tabular}


Table 13. The performance experimental results of deep learning approaches relative in Dataset_2_class (Binary classification).

\begin{tabular}{cccc}
\hline & DNN & RNN & CNN \\
\hline TNR (BENIGN) & $96 \%$ & $99 \%$ & $99 \%$ \\
\hline Attack & $100 \%$ & $100 \%$ & $100 \%$ \\
\hline
\end{tabular}

The performance experimental results of deep learning approaches in term of F-score with binary classification and multiclass classification are presented in Figure 9. The results show that deep learning techniques can provide better performance in cyber security intrusion detection for Agriculture 4.0. The Receiver Operating Characteristic (ROC) curve for Dataset_13_class is depicted in Figure 10, which is a plot of intrusion detection accuracy against the false positive probability. We can identify the obvious better performance of both deep learning techniques, namely, convolutional neural network and recurrent neural network, since all the values of AUC (Area Under Curve) for three classes, including, class 0: BENIGN, class 1: DrDoS_DNS, and class 2: DrDoS_LDAP, are between 0.94 and 1.00.

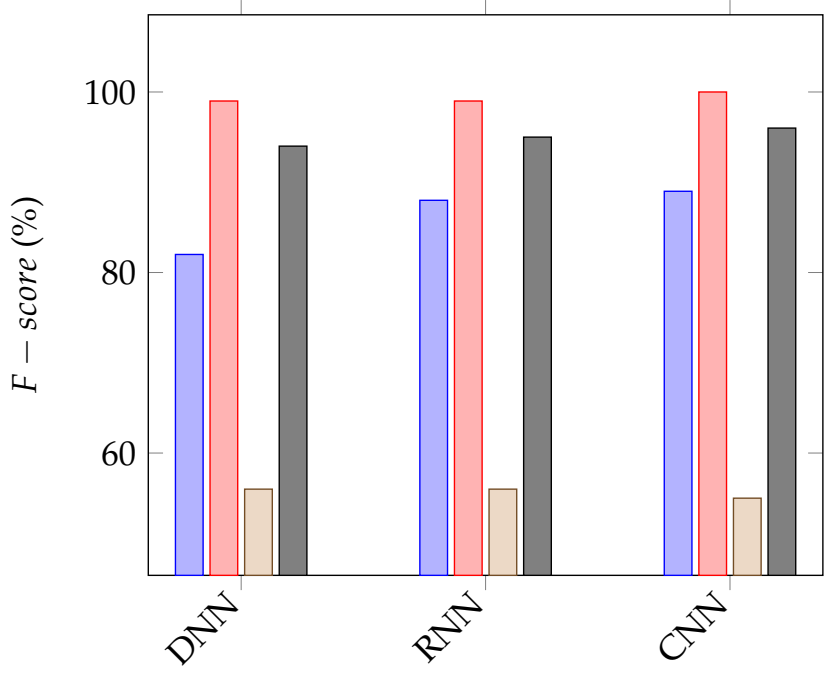

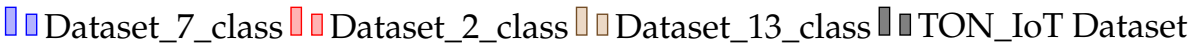

Figure 9. The performance experimental results of deep learning approaches in term of F-score with binary classification and multiclass classification.

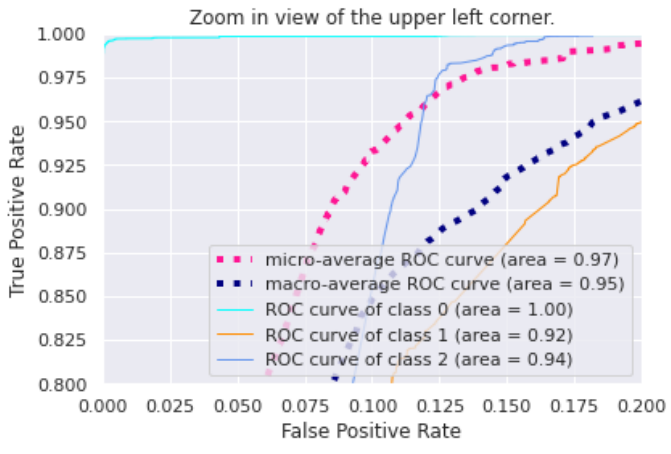

(a) Convolutional neural network (CNN)

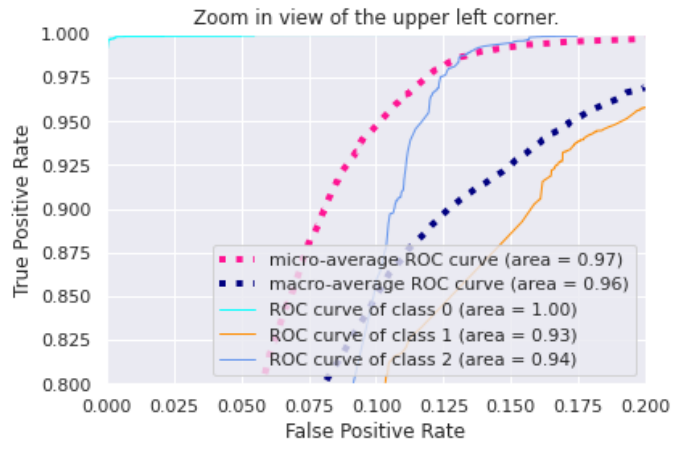

(b) Recurrent neural network (RNN)

Figure 10. The Receiver Operating Characteristic (ROC) for Dataset_13_class. class 0: BENIGN, class 1: DrDoS_DNS, class 2: DrDoS_LDAP.

Table 14 presents the accuracy, FAR, and training time of deep learning approaches with different hidden nodes and learning rates in four datasets; namely, Dataset_2_class, 
Dataset_7_class, Dataset_13_class, and TON_IoT dataset. In binary class classification (i.e., Dataset_2_class) and TON_IoT dataset, the convolutional neural network achieves high accuracy of $99.95 \%$ compared to both recurrent neural network and deep neural network, when the number of hidden nodes is 100 and the learning rate is 0.5. In multi-class classification (i.e., Dataset_7_class), the recurrent neural network achieves high accuracy of $93.88 \%$ compared to both deep neural network and convolutional neural network, when the learning rate is 0.5 and the number of hidden nodes is 30 . In multi-class classification (i.e., Dataset_13_class), the convolutional neural network achieves high accuracy of $95.12 \%$ compared to both recurrent neural network and deep neural network, when the number of hidden nodes is 100 and the learning rate is 0.5 . These results show that the recurrent neural network is efficient compared to the convolutional neural network with a lower number of hidden nodes.

Table 14. The accuracy, FAR, and training time of deep learning approaches with different hidden nodes and learning rate in four datasets, namely, Dataset_2_class, Dataset_7_class, Dataset_13_class, and TON_IoT dataset.

\begin{tabular}{|c|c|c|c|c|c|c|c|c|c|c|c|c|c|}
\hline \multirow{2}{*}{ Parameters } & \multirow{2}{*}{$\begin{array}{l}\text { Performance } \\
\text { Metrics }\end{array}$} & \multicolumn{3}{|c|}{ Dataset_2_Class } & \multicolumn{3}{|c|}{ Dataset_7_Class } & \multicolumn{3}{|c|}{ Dataset_13_Class } & \multicolumn{3}{|c|}{ TON_IoT Dataset } \\
\hline & & DNN & RNN & $\mathrm{CNN}$ & DNN & RNN & $\mathrm{CNN}$ & DNN & RNN & CNN & DNN & RNN & $\mathrm{CNN}$ \\
\hline \multirow{3}{*}{$\begin{array}{c}H N=30 \\
L R=0.01\end{array}$} & ACC & $99.92 \%$ & $99.93 \%$ & $99.90 \%$ & $93.53 \%$ & $93.88 \%$ & $93.48 \%$ & $75.26 \%$ & $78.29 \%$ & $72.28 \%$ & $98.91 \%$ & $98.92 \%$ & $98.89 \%$ \\
\hline & FAR & $1.14 \%$ & $1.13 \%$ & $1.15 \%$ & $2.14 \%$ & $2.12 \%$ & $2.15 \%$ & $3.14 \%$ & $3.11 \%$ & $3.16 \%$ & $1.12 \%$ & $1.11 \%$ & $1.13 \%$ \\
\hline & Time & 31 & 60 & 30 & 122 & 142 & 120 & 193 & 211 & 181 & 33 & 63 & 34 \\
\hline \multirow{3}{*}{$\begin{array}{l}H N=30 \\
L R=0.1\end{array}$} & ACC & $99.92 \%$ & $99.93 \%$ & $99.90 \%$ & $93.53 \%$ & $93.88 \%$ & $93.48 \%$ & $75.26 \%$ & $78.29 \%$ & $72.28 \%$ & $98.02 \%$ & $98.03 \%$ & $98.00 \%$ \\
\hline & FAR & $1.14 \%$ & $1.13 \%$ & $1.15 \%$ & $2.14 \%$ & $2.12 \%$ & $2.15 \%$ & $3.14 \%$ & $3.11 \%$ & $3.16 \%$ & $1.13 \%$ & $1.12 \%$ & $1.14 \%$ \\
\hline & Time & 34 & 66 & 35 & 134 & 123 & 125 & 199 & 223 & 191 & 35 & 67 & 36 \\
\hline \multirow{3}{*}{$\begin{array}{l}H N=30 \\
L R=0.5\end{array}$} & ACC & $99.92 \%$ & $99.93 \%$ & $99.90 \%$ & $93.53 \%$ & $93.88 \%$ & $93.48 \%$ & $75.26 \%$ & $78.29 \%$ & $72.28 \%$ & $98.92 \%$ & $98.91 \%$ & $98.81 \%$ \\
\hline & FAR & $1.14 \%$ & $1.13 \%$ & $1.15 \%$ & $2.14 \%$ & $2.12 \%$ & $2.15 \%$ & $3.14 \%$ & $3.11 \%$ & $3.16 \%$ & $1.15 \%$ & $1.14 \%$ & $1.16 \%$ \\
\hline & Time & 38 & 69 & 39 & 138 & 128 & 130 & 211 & 228 & 196 & 49 & 79 & 48 \\
\hline \multirow{3}{*}{$\begin{array}{c}H N=60 \\
L R=0.01\end{array}$} & ACC & $99.93 \%$ & $99.94 \%$ & $99.94 \%$ & $93.53 \%$ & $93.88 \%$ & $93.89 \%$ & $75.99 \%$ & $78.29 \%$ & $78.92 \%$ & $98.92 \%$ & $98.93 \%$ & $98.83 \%$ \\
\hline & FAR & $1.14 \%$ & $1.13 \%$ & $1.13 \%$ & $2.14 \%$ & $2.12 \%$ & $2.08 \%$ & $3.02 \%$ & $2.11 \%$ & $2.08 \%$ & $1.20 \%$ & $1.18 \%$ & $1.19 \%$ \\
\hline & Time & 32 & 61 & 31 & 123 & 144 & 122 & 194 & 214 & 183 & 52 & 71 & 94 \\
\hline \multirow{3}{*}{$\begin{array}{l}H N=60 \\
L R=0.1\end{array}$} & ACC & $99.93 \%$ & $99.94 \%$ & $99.94 \%$ & $93.53 \%$ & $93.88 \%$ & $93.89 \%$ & $75.99 \%$ & $78.29 \%$ & $78.92 \%$ & $98.90 \%$ & $98.93 \%$ & $98.90 \%$ \\
\hline & FAR & $1.14 \%$ & $1.13 \%$ & $1.13 \%$ & $2.14 \%$ & $2.12 \%$ & $2.08 \%$ & $3.02 \%$ & $2.11 \%$ & $2.08 \%$ & $1.29 \%$ & $1.23 \%$ & $1.24 \%$ \\
\hline & Time & 39 & 63 & 34 & 126 & 147 & 125 & 199 & 217 & 186 & 79 & 93 & 94 \\
\hline \multirow{3}{*}{$\begin{array}{l}H N=60 \\
L R=0.5\end{array}$} & ACC & $99.93 \%$ & $99.94 \%$ & $99.95 \%$ & $93.53 \%$ & $93.88 \%$ & $93.90 \%$ & $75.99 \%$ & $78.29 \%$ & $80.02 \%$ & $98.93 \%$ & $98.94 \%$ & $98.94 \%$ \\
\hline & FAR & $1.14 \%$ & $1.13 \%$ & $1.10 \%$ & $2.14 \%$ & $2.12 \%$ & $2.05 \%$ & $3.02 \%$ & $2.11 \%$ & $2.08 \%$ & $1.94 \%$ & $1.93 \%$ & $1.90 \%$ \\
\hline & Time & 42 & 69 & 40 & 140 & 130 & 132 & 199 & 217 & 186 & 72 & 89 & 80 \\
\hline \multirow{3}{*}{$\begin{array}{l}H N=100 \\
L R=0.01\end{array}$} & ACC & $99.93 \%$ & $99.94 \%$ & $99.94 \%$ & $94.52 \%$ & $94.89 \%$ & $94.91 \%$ & $85.99 \%$ & $88.22 \%$ & $90.99 \%$ & $98.93 \%$ & $98.94 \%$ & $98.94 \%$ \\
\hline & FAR & $1.14 \%$ & $1.13 \%$ & $1.13 \%$ & $1.99 \%$ & $1.80 \%$ & $1.78 \%$ & $2.22 \%$ & $2.08 \%$ & $2.01 \%$ & $1.94 \%$ & $1.83 \%$ & $1.73 \%$ \\
\hline & Time & 42 & 72 & 42 & 123 & 144 & 132 & 222 & 250 & 241 & 82 & 92 & 94 \\
\hline \multirow{3}{*}{$\begin{aligned} H N & =100 \\
L R & =0.1\end{aligned}$} & ACC & $99.93 \%$ & $99.94 \%$ & $99.94 \%$ & $94.52 \%$ & $94.89 \%$ & $94.91 \%$ & $89.99 \%$ & $91.32 \%$ & $92.24 \%$ & $98.93 \%$ & $98.95 \%$ & $98.95 \%$ \\
\hline & FAR & $1.14 \%$ & $1.13 \%$ & $1.13 \%$ & $1.99 \%$ & $1.80 \%$ & $1.78 \%$ & $2.04 \%$ & $2.01 \%$ & $1.90 \%$ & $1.84 \%$ & $1.73 \%$ & $1.72 \%$ \\
\hline & Time & 60 & 102 & 80 & 152 & 170 & 182 & 231 & 282 & 271 & 90 & 129 & 92 \\
\hline \multirow{3}{*}{$\begin{aligned} H N & =100 \\
L R & =0.5\end{aligned}$} & ACC & $99.93 \%$ & $99.94 \%$ & $99.95 \%$ & $94.91 \%$ & $94.99 \%$ & $95.90 \%$ & $93.98 \%$ & $94.88 \%$ & $95.12 \%$ & $98.93 \%$ & $98.94 \%$ & $99.92 \%$ \\
\hline & FAR & $1.14 \%$ & $1.13 \%$ & $1.10 \%$ & $1.80 \%$ & $1.78 \%$ & $1.50 \%$ & $2.02 \%$ & $1.99 \%$ & $1.77 \%$ & $1.94 \%$ & $1.82 \%$ & $0.80 \%$ \\
\hline & Time & 102 & 151 & 120 & 180 & 191 & 221 & 252 & 302 & 311 & 172 & 261 & 220 \\
\hline
\end{tabular}

Table 15 compares the performance of our work with other state-of-the-art methods that are tested under the CIC-DDoS2019 dataset and the TON_IoT dataset. The comparison is conducted with respect to network model, dataset, task, machine learning model, and accuracy. We can observe that our IDS model based on CNN incur the best results in terms of accuracy. This is due to the strategy of our pre-processing for both datasets that reduce the computation complexity and due to the use of simple deep learning models with larger batch sizes and fewer layers. 
Table 15. Comparison with related work tested on CIC-DDoS2019 dataset and TON_IoT dataset.

\begin{tabular}{|c|c|c|c|c|c|c|}
\hline IDS Model & Year & Network Model & Dataset & Task & Model & Accuracy \\
\hline Jia et al. [39] & 2020 & IoT application & CIC-DDoS2019 dataset & Multiclass (13 class) & LSTM & $98.9 \%$ \\
\hline Li et al. [40] & 2020 & IoT application & CIC-DDoS2019 dataset & Multiclass (13 class) & LSTM & $\mathrm{N} / \mathrm{A}$ \\
\hline de Assis et al. [41] & 2020 & $\begin{array}{l}\text { SDN environments in IoT net- } \\
\text { works }\end{array}$ & CIC-DDoS2019 dataset & Multiclass (13 class) & $\mathrm{CNN}$ & $95.4 \%$ \\
\hline Alamri et al. [42] & 2020 & $\begin{array}{l}\text { SDN environments in IoT net- } \\
\text { works }\end{array}$ & CIC-DDoS2019 dataset & Multiclass (13 class) & $\begin{array}{l}\text { Extreme gradient } \\
\text { boosting algorithm }\end{array}$ & $91.26 \%$ \\
\hline Zhang et al. [43] & 2020 & $\begin{array}{l}\text { SDN environments in IoT net- } \\
\text { works }\end{array}$ & TON_IoT dataset & Multiclass & Random Forest & $99.68 \%$ \\
\hline Kumar et al. [44] & 2021 & IoT application & TON_IoT dataset & Multiclass & $\begin{array}{l}\text { Extreme gradient } \\
\text { boosting algorithm }\end{array}$ & $97.45 \%$ \\
\hline Pontes et al. [45] & 2021 & $\mathrm{~N} / \mathrm{A}$ & CIC-DDoS2019 dataset & Multiclass (13 class) & $\begin{array}{l}\text { Energy-based flow } \\
\text { classifier }\end{array}$ & $98.1 \%$ \\
\hline \multirow{2}{*}{ Assis et al. [46] } & \multirow[b]{2}{*}{2021} & \multirow{2}{*}{ SDN environments } & \multirow{2}{*}{ CIC-DDoS2019 dataset } & Binary ( 2 class) & \multirow{2}{*}{$\begin{array}{l}\text { Gated Recurrent } \\
\text { Units (GRU) }\end{array}$} & $99.6 \%$ \\
\hline & & & & Multiclass (13 class) & & $\sim 99 \%$ \\
\hline Kumar et al. [47] & 2021 & IoT application & TON_IoT dataset & Multiclass & $\begin{array}{l}\text { Extreme gradient } \\
\text { boosting algorithm }\end{array}$ & $96.35 \%$ \\
\hline Javeed et al. [48] & 2021 & $\begin{array}{l}\text { SDN environments in IoT net- } \\
\text { works }\end{array}$ & CIC-DDoS2019 dataset & Multiclass (13 class) & LSTM and GRU & $99.74 \%$ \\
\hline Nie et al. [49] & 2021 & IoT application & CIC-DDoS2019 dataset & Multiclass (13 class) & $\begin{array}{l}\text { Generative adver- } \\
\text { sarial network }\end{array}$ & $98.35 \%$ \\
\hline Kumar et al. [50] & 2021 & $\begin{array}{l}\text { Smart agricultural Unmanned } \\
\text { Aerial Vehicles }\end{array}$ & TON_IoT dataset & Multiclass & $\begin{array}{l}\text { Stacked Long-Short- } \\
\text { Term Memory }\end{array}$ & $88.82 \%$ \\
\hline \multirow{12}{*}{ Our model } & \multirow{12}{*}{-} & \multirow{12}{*}{$\begin{array}{l}\text { Agriculture } 4.0 \text { based } \\
\text { on the following IoT } \\
\text { technologies, } \\
\text { including, IoT devices, } \\
5 \mathrm{G} \text { communications, } \\
\text { Drones,Fog/Edge computing, } \\
\text { Cloud Computing, } \\
\text { NFV, and SDN }\end{array}$} & \multirow{9}{*}{ CIC-DDoS2019 dataset } & \multirow{3}{*}{ Binary ( 2 class) } & $\mathrm{CNN}$ & $99.95 \%$ \\
\hline & & & & & RNN & $99.94 \%$ \\
\hline & & & & & DNN & $99.93 \%$ \\
\hline & & & & \multirow{3}{*}{ Multiclass (7 class) } & $\mathrm{CNN}$ & $95.90 \%$ \\
\hline & & & & & RNN & $94.99 \%$ \\
\hline & & & & & DNN & $94.91 \%$ \\
\hline & & & & \multirow{3}{*}{ Multiclass (13 class) } & $\mathrm{CNN}$ & $95.12 \%$ \\
\hline & & & & & RNN & $94.88 \%$ \\
\hline & & & & & DNN & $93.88 \%$ \\
\hline & & & \multirow{3}{*}{ TON_IoT dataset } & \multirow{3}{*}{ Multiclass } & $\mathrm{CNN}$ & $99.92 \%$ \\
\hline & & & & & RNN & $98.94 \%$ \\
\hline & & & & & DNN & $98.93 \%$ \\
\hline
\end{tabular}

\section{Conclusions}

In this paper, we proposed three deep learning-based IDS models, including a convolutional neural network-based IDS model, a deep neural network-based IDS model, and a recurrent neural network-based IDS model. Specifically, we provided a performance evaluation and comparative analysis of machine learning and deep learning approaches for cyber security in agriculture 4.0. Each model's performance is studied within two classification types (binary and multiclass) using two new real traffic datasets; namely, CICDDoS2019 dataset and TON_IoT dataset. The results show that deep learning techniques give good results in comparison to other machine learning strategies (e.g., decision tree, random forests, naive bayes, and logistic regression) in terms of important performance indicators, including detection rate, false alarm rate, precision, F-score, recall, true negative rate, false accept rate, ROC Curve, and accuracy. In addition, the IDS model based on CNN outperforms the state-of-the-art deep learning IDS methods, which were tested under the CIC-DDoS2019 dataset and TON_IoT dataset, by recording an accuracy of $99.95 \%$ for binary traffic detection and $99.92 \%$ for multiclass traffic detection.

Author Contributions: Conceptualization, M.A.F., L.S., H.D. and K.-K.R.C.; Methodology, M.A.F., L.S., H.D. and K.-K.R.C.; Software, M.A.F., L.S., and H.D.; Validation, M.A.F., L.S., and H.D.; formal analysis, M.A.F., L.S., and H.D.; investigation, M.A.F., L.S., and H.D.; resources, M.A.F., L.S., and H.D.; data curation, M.A.F., L.S., and H.D.; writing-original draft preparation, M.A.F., L.S., and H.D.; writing-review and editing, M.A.F., L.S., and K.-K.R.C.; visualization, M.A.F., L.S.; supervision, M.A.F., L.S. and K.-K.R.C. All authors have read and agreed to the published version of the manuscript.

Funding: This work was supported in part by the Research Start-Up Fund for Talent Researcher of Nanjing Agricultural University under Grant 77H0603 and in part by the National Natural Science 
Foundation of China under Grant 62072248. The work of K.-K. R. Choo was supported only by the Cloud Technology Endowed Professorship.

Data Availability Statement: We used the CIC-DDoS2019 dataset and TON_IoT dataset, which are publicly accessed datasets (https:/ / www.unb.ca/cic/datasets/ddos-2019.html, https: / /ieeedataport.org/documents/toniot-datasets) (accessed on 1 April 2021), for the evaluation of the proposed IDS.

Conflicts of Interest: All authors declare no conflict of interest.

\section{References}

1. Chen, B.; Wan, J.; Shu, L.; Li, P.; Mukherjee, M.; Yin, B. Smart factory of industry 4.0: Key technologies, application case, and challenges. IEEE Access 2017, 6, 6505-6519. [CrossRef]

2. Friha, O.; Ferrag, M.A.; Shu, L.; Maglaras, L.; Wang, X. Internet of Things for the Future of Smart Agriculture: A Comprehensive Survey of Emerging Technologies. IEEE/CAA J. Autom. Sin. 2021, 8, 718-752. [CrossRef]

3. Liu, Y.; Ma, X.; Shu, L.; Hancke, G.P.; Abu-Mahfouz, A.M. From Industry 4.0 to Agriculture 4.0: Current Status, Enabling Technologies, and Research Challenges. IEEE Trans. Ind. Inform. 2020, 17, 4322-4334.

4. Ferrag, M.A.; Shu, L.; Yang, X.; Derhab, A.; Maglaras, L. Security and Privacy for Green IoT-Based Agriculture: Review, Blockchain Solutions, and Challenges. IEEE Access 2020, 8, 32031-32053. [CrossRef]

5. Yang, X.; Shu, L.; Chen, J.; Ferrag, M.A.; Wu, J.; Nurellari, E.; Huang, K. A Survey on Smart Agriculture: Development Modes, Technologies, and Security and Privacy Challenges. IEEE/CAA J. Autom. Sin. 2021, 8, 273-302. [CrossRef]

6. Buczak, A.L.; Guven, E. A survey of data mining and machine learning methods for cyber security intrusion detection. IEEE Commun. Surv. Tutor. 2015, 18, 1153-1176. [CrossRef]

7. Chen, J.W.; Lin, W.J.; Cheng, H.J.; Hung, C.L.; Lin, C.Y.; Chen, S.P. A smartphone-based application for scale pest detection using multiple-object detection methods. Electronics 2021, 10, 372. [CrossRef]

8. Liao, H.J.; Lin, C.H.R.; Lin, Y.C.; Tung, K.Y. Intrusion detection system: A comprehensive review. J. Netw. Comput. Appl. 2013, 36, 16-24. [CrossRef]

9. Muna, A.H.; Sitnikova, E. Developing a Security Testbed for Industrial Internet of Things. IEEE Internet Things J. 2020, 8 , 5558-5573.

10. Kasongo, S.M.; Sun, Y. A deep learning method with wrapper based feature extraction for wireless intrusion detection system. Comput. Secur. 2020, 92, 101752. [CrossRef]

11. Hassan, M.M.; Gumaei, A.; Alsanad, A.; Alrubaian, M.; Fortino, G. A hybrid deep learning model for efficient intrusion detection in big data environment. Inf. Sci. 2020, 513, 386-396. [CrossRef]

12. Li, B.; Wu, Y.; Song, J.; Lu, R.; Li, T.; Zhao, L. DeepFed: Federated Deep Learning for Intrusion Detection in Industrial Cyber-Physical Systems. IEEE Trans. Ind. Inform. 2020, 17, 5615-5624. [CrossRef]

13. Gao, J.; Gan, L.; Buschendorf, F.; Zhang, L.; Liu, H.; Li, P.; Dong, X.; Lu, T. Omni SCADA intrusion detection using deep learning algorithms. IEEE Internet Things J. 2020, 8, 951-961. [CrossRef]

14. Ferrag, M.A.; Maglaras, L. DeepCoin: A novel deep learning and blockchain-based energy exchange framework for smart grids. IEEE Trans. Eng. Manag. 2019, 67, 1285-1297. [CrossRef]

15. Nie, L.; Ning, Z.; Wang, X.; Hu, X.; Li, Y.; Cheng, J. Data-Driven Intrusion Detection for Intelligent Internet of Vehicles: A Deep Convolutional Neural Network-based Method. IEEE Trans. Netw. Sci. Eng. 2020, 7, 2219-2230. [CrossRef]

16. Abusitta, A.; Bellaiche, M.; Dagenais, M.; Halabi, T. A deep learning approach for proactive multi-cloud cooperative intrusion detection system. Future Gener. Comput. Syst. 2019, 98, 308-318. [CrossRef]

17. Kamilaris, A.; Prenafeta-Boldú, F.X. Deep learning in agriculture: A survey. Comput. Electron. Agric. 2018, 147, 70-90. [CrossRef]

18. Top 8 Challenges for Machine Learning Practitioners. Available online: https://towardsdatascience.com/top-8-challenges-formachine-learning-practitioners-c4c0130701a1 (accessed on 1 May 2021).

19. Ferrag, M.A.; Maglaras, L.; Janicke, H.; Smith, R. Deep learning techniques for cyber security intrusion detection: A detailed analysis. In Proceedings of the 6th International Symposium for ICS \& SCADA Cyber Security Research 2019, Athens, Greece, 10-12 September 2019; pp. 126-136.

20. Diro, A.A.; Chilamkurti, N. Distributed attack detection scheme using deep learning approach for Internet of Things. Future Gener. Comput. Syst. 2018, 82, 761-768. [CrossRef]

21. Muna, A.H.; Moustafa, N.; Sitnikova, E. Identification of malicious activities in industrial internet of things based on deep learning models. J. Inf. Secur. Appl. 2018, 41, 1-11.

22. HaddadPajouh, H.; Dehghantanha, A.; Khayami, R.; Choo, K.K.R. A deep recurrent neural network based approach for internet of things malware threat hunting. Future Gener. Comput. Syst. 2018, 85, 88-96. [CrossRef]

23. Vinayakumar, R.; Alazab, M.; Srinivasan, S.; Pham, Q.V.; Padannayil, S.K.; Simran, K. A visualized botnet detection system based deep learning for the Internet of Things networks of smart cities. IEEE Trans. Ind. Appl. 2020, 56, 4436-4456. [CrossRef]

24. Parra, G.D.L.T.; Rad, P.; Choo, K.K.R.; Beebe, N. Detecting Internet of Things attacks using distributed deep learning. J. Netw. Comput. Appl. 2020, 163, 102662. [CrossRef] 
25. Latif, S.; Zou, Z.; Idrees, Z.; Ahmad, J. A Novel Attack Detection Scheme for the Industrial Internet of Things Using a Lightweight Random Neural Network. IEEE Access 2020, 8, 89337-89350. [CrossRef]

26. Manimurugan, S.; Al-Mutairi, S.; Aborokbah, M.M.; Chilamkurti, N.; Ganesan, S.; Patan, R. Effective Attack Detection in Internet of Medical Things Smart Environment Using a Deep Belief Neural Network. IEEE Access 2020, 8, 77396-77404. [CrossRef]

27. Koroniotis, N.; Moustafa, N.; Sitnikova, E. A new network forensic framework based on deep learning for Internet of Things networks: A particle deep framework. Future Gener. Comput. Syst. 2020, 110, 91-106. [CrossRef]

28. Zhou, X.; Hu, Y.; Liang, W.; Ma, J.; Jin, Q. Variational LSTM enhanced anomaly detection for industrial big data. IEEE Trans. Ind. Inform. 2020, 17, 3469-3477. [CrossRef]

29. Bhuvaneswari Amma, N.G.; Selvakumar, S. Anomaly detection framework for Internet of things traffic using vector convolutional deep learning approach in fog environment. Future Gener. Comput. Syst. 2020, 113, 255-265.

30. Khoa, T.V.; Saputra, Y.M.; Hoang, D.T.; Trung, N.L.; Nguyen, D.; Ha, N.V.; Dutkiewicz, E. Collaborative learning model for cyberattack detection systems in iot industry 4.0. In Proceedings of the 2020 IEEE Wireless Communications and Networking Conference (WCNC). Seoul, Korea, 25-28 May 2020; pp. 1-6.

31. Popoola, S.I.; Adebisi, B.; Hammoudeh, M.; Gui, G.; Gacanin, H. Hybrid Deep Learning for Botnet Attack Detection in the Internet of Things Networks. IEEE Internet Things J. 2021, 8, 4944-4956. [CrossRef]

32. Al-Hawawreh, M.; Moustafa, N.; Garg, S.; Hossain, M.S. Deep Learning-enabled Threat Intelligence Scheme in the Internet of Things Networks. IEEE Trans. Netw. Sci. Eng. 2020. [CrossRef]

33. Ge, M.; Syed, N.F.; Fu, X.; Baig, Z.; Robles-Kelly, A. Towards a deep learning-driven intrusion detection approach for Internet of Things. Comput. Netw. 2021, 186, 107784. [CrossRef]

34. Sharafaldin, I.; Lashkari, A.H.; Hakak, S.; Ghorbani, A.A. Developing realistic distributed denial of service (DDoS) attack dataset and taxonomy. In Proceedings of the 2019 International Carnahan Conference on Security Technology (ICCST), Chennai, India, 1-3 October 2019; pp. 1-8.

35. TON_IOT DATASETS. Available online: https://ieee-dataport.org/documents/toniot-datasets (accessed on 4 April 2021).

36. DiPietro, R.; Hager, G.D. Deep learning: RNNs and LSTM. In Handbook of Medical Image Computing and Computer Assisted Intervention; Elsevier: Amsterdam, The Netherlands, 2020; pp. 503-519.

37. LeCun, Y.; Bengio, Y.; Hinton, G. Deep learning. Nature 2015, 521, 436-444. [CrossRef] [PubMed]

38. Alsaedi, A.; Moustafa, N.; Tari, Z.; Mahmood, A.; Anwar, A. TON_IoT telemetry dataset: A new generation dataset of IoT and IIoT for data-driven Intrusion Detection Systems. IEEE Access 2020, 8, 165130-165150. [CrossRef]

39. Jia, Y.; Zhong, F.; Alrawais, A.; Gong, B.; Cheng, X. Flowguard: An intelligent edge defense mechanism against IoT DDoS attacks. IEEE Internet Things J. 2020, 7, 9552-9562. [CrossRef]

40. Li, J.; Liu, M.; Xue, Z.; Fan, X.; He, X. Rtvd: A real-time volumetric detection scheme for ddos in the internet of things. IEEE Access 2020, 8, 36191-36201. [CrossRef]

41. de Assis, M.V.; Carvalho, L.F.; Rodrigues, J.J.; Lloret, J.; Proença, M.L., Jr. Near real-time security system applied to SDN environments in IoT networks using convolutional neural network. Comput. Electr. Eng. 2020, 86, 106738. [CrossRef]

42. Alamri, H.A.; Thayananthan, V. Bandwidth Control Mechanism and Extreme Gradient Boosting Algorithm for Protecting Software-Defined Networks Against DDoS Attacks. IEEE Access 2020, 8, 194269-194288. [CrossRef]

43. Zhang, Y.; Xu, J.; Wang, Z.; Geng, R.; Choo, K.K.R.; Pérez-Díaz, J.A.; Zhu, D. Efficient and Intelligent Attack Detection in Software Defined IoT Networks. In Proceedings of the 2020 IEEE International Conference on Embedded Software and Systems (ICESS), Shanghai, China, 10-11 December 2020; pp. 1-9.

44. Kumar, P.; Gupta, G.P.; Tripathi, R. TP2SF: A Trustworthy Privacy-Preserving Secured Framework for sustainable smart cities by leveraging blockchain and machine learning. J. Syst. Archit. 2021, 115, 101954. [CrossRef]

45. Pontes, C.; Souza, M.; Gondim, J.; Bishop, M.; Marotta, M. A new method for flow-based network intrusion detection using the inverse Potts model. IEEE Trans. Netw. Serv. Manag. 2021. [CrossRef]

46. Assis, M.V.; Carvalho, L.F.; Lloret, J.; Proença, M.L., Jr. A GRU deep learning system against attacks in software defined networks. J. Netw. Comput. Appl. 2021, 177, 102942. [CrossRef]

47. Kumar, P.; Gupta, G.P.; Tripathi, R. An ensemble learning and fog-cloud architecture-driven cyber-attack detection framework for IoMT networks. Comput. Commun. 2021, 166, 110-124. [CrossRef]

48. Javeed, D.; Gao, T.; Khan, M.T. SDN-Enabled Hybrid DL-Driven Framework for the Detection of Emerging Cyber Threats in IoT. Electronics 2021, 10, 918. [CrossRef]

49. Nie, L.; Wu, Y.; Wang, X.; Guo, L.; Wang, G.; Gao, X.; Li, S. Intrusion Detection for Secure Social Internet of Things Based on Collaborative Edge Computing: A Generative Adversarial Network-Based Approach. IEEE Trans. Comput. Soc. Syst. 2021. [CrossRef]

50. Kumar, R.; Kumar, P.; Tripathi, R.; Gupta, G.P.; Gadekallu, T.R.; Srivastava, G. Sp2f: A secured privacy-preserving framework for smart agricultural unmanned aerial vehicles. Comput. Netw. 2021, 187, 107819. [CrossRef] 